\title{
Delivery of Splice Switching Oligonucleotides by Amphiphilic Chitosan-Based Nanoparticles
}

\section{Pedro M. D. Moreno ${ }^{\star \dagger}$, Joyce C. Santos ${ }^{\dagger \ddagger \S}$, Carla P. Gomes $^{+\ddagger /}$, Aida Varela-Moreira ${ }^{\dagger \ddagger} \perp$, Artur Costa ${ }^{\dagger \ddagger}$, Victoria Leiro ${ }^{\ddagger \neq}$, Herman Mansur§, Ana P. Pêgo ${ }^{\star^{\ddagger \neq / /} \nabla}$}

† INEB-Instituto de Engenharia Biomédica, Universidade do Porto (UPorto), Rua Alfredo Allen, 208, 4200-135 Porto, Portugal

\# i3S-Instituto de Investigação e Inovação em Saúde, UPorto, 4200-135 Porto, Portugal $\int \mathrm{CeNano2}$ I, Department of Metallurgical and Materials Engineering, UFMG, 31270-901 Belo Horizonte, Brazil

// Faculdade de Engenharia da UPorto (FEUP), 4200-319 Porto, Portugal

$\perp$ Faculdade de Medicina da UPorto (FMUP), 4200-319 Porto, Portugal

$\nabla$ Instituto de Ciências Biomédicas Abel Salazar (ICBAS), UPorto, 4050-313 Porto, Portugal

*Telephone: +351-701-231-7943; fax: +351-701-231-8333; e-mail: Pedro M.D. Moreno (pedro.moreno@ineb.up.pt).

*Telephone: +351-701-231-7943; fax: +351-701-231-8333; e-mail: Ana P. Pêgo (apego@ineb.up.pt).

Originally published in Mol. Pharmaceutics 2016, 13, 2, 344-356 https://doi.org/10.1021/acs.molpharmaceut. 5600538 


\section{ABSTRACT}

Splice switching oligonucleotides (SSOs) are a class of single-stranded antisense oligonucleotides (ssONs) being used as gene therapeutics and demonstrating great therapeutic potential. The availability of biodegradable and biocompatible delivery vectors that could improve delivery efficiencies, reduce dosage, and, in parallel, reduce toxicity concerns could be advantageous for clinical translation. In this work we explored the use of quaternized amphiphilic chitosan-based vectors in nanocomplex formation and delivery of splice switching oligonucleotides (SSO) into cells, while providing insights regarding cellular uptake of such complexes. Results show that the chitosan amphiphilic character is important when dealing with SSOs, greatly improving colloidal stability under serum conditions, as analyzed by dynamic light scattering, and enhancing cellular association. Nanocomplexes were found to follow an endolysosomal route with a long lysosome residence time. Conjugation of a hydrophobic moiety, stearic acid, to quaternized chitosan was a necessary condition to achieve transfection, as an unmodified quaternary chitosan was completely ineffective. We thus demonstrate that amphiphilic quaternized chitosan is a biomaterial that holds promise and warrants further development as a platform for SSO delivery strategies.

KEYWORDS: chitosan, trimethylchitosan, hydrophobic, nanoparticles, splice switching oligonucleotides

\section{INTRODUCTION}

Therapeutic antisense oligonucleotides (AONs) hold great potential for the treatment of different disease conditions. Especially promising are splice switching oligonucleotides (SSOs). These singlestranded antisense oligonucleotides ( $\mathrm{s} \mathrm{SONs}$ ) have seen enormous development as potential pharmaceuticals, which is attributed to the fact that more than $95 \%$ of human pre-mRNA undergoes alternative splicing(1) and, especially important, a variety of cancers and genetic diseases have been linked to alternative splicing aberrations. $(2,3)$ Mechanistically, SSOs act by specifically binding to pre-mRNA in the nucleus and sterically hindering the access of the splicing machinery proteins to the pre-mRNA, thereby promoting alteration of splicing patterns.(4) Highly promising results have been shown in different areas such as muscular, $(5)$ neuromuscular, $(6)$ immunological, $(7,8)$ cancer, $(9,10)$ metabolic,(11) and blood(12) disorders. Recent advances in nucleic acid chemistry have enhanced both intracellular RNA target recognition and resistance to degradation, allowing the in vivo delivery of free SSO. Nonetheless, the cellular uptake processes of naked SSOs are still rather ineffective,(13) warranting the development of vectorization systems.

An interesting vector known for its biocompatibility, low cytotoxicity, and biodegradability is chitosan. This is a natural linear copolymer of d-glucosamine and $\mathrm{N}$-acetyl-d-glucosamine in a $\beta$-(14) linkage, whose properties are interdependent on the degree of polymerization (i.e., molecular weight) and ratio between the repeating units (i.e., degree of acetylation, DA). Chitosan has a cationic character under mildly acidic conditions due to protonation of its primary amines at $\mathrm{pH}$ lower than its $p K_{a}$, often reported to be around 6.5 , although it can slightly vary according to the degree of acetylation and ionic strength conditions.(14) This cationic character has thus been used for the formation of polyelectrolyte complexes with negatively charged nucleic acids aiming at cellular delivery.(15) While chitosan and several chitosan derivatives have been carefully studied regarding 
complexation with pDNA and siRNA molecules, and also factors affecting delivery efficiencies, there are limited reports on their use with ssONs and none specifically dealing with SSOs.(16-21) Concurrently, complexation of SSOs (and ssONs in general) with polycationic vectors poses an additional challenge, as their small sizes result in low electrostatic binding forces taking place (lower than for siRNA). Moreover, despite some flexibility of the single-stranded structure, ssONs are unsuitable for "condensation".

Thus, development of improved chitosan-based vectors for enhanced complexation and delivery of SSOs is warranted. Additionally, a more comprehensive view on complex stability and cellular interactions is still lacking.

To this end, we have here explored quaternization and hydrophobic modification of the chitosan backbone. First, quaternization by methylation of primary amine groups confers to the polymer a permanent positive charge which enhances both electrostatic interactions during complex formation and solubility at neutral pH. (22) This is especially important, as chitosan depends on acidic conditions for its protonation, meaning that, under physiological conditions, the surface charge and colloidal stability of complexes is reduced, making the particles unstable and prone to aggregation. Second, hydrophobization can lead to improved intra- and intermolecular polymer chain interactions, resulting in self-assembly and enhanced colloidal stability of chitosan-nucleic acid complexes. In addition, the effect of hydrophobic modifications on nucleic acids delivery has been associated with increased cell membrane interactions of the complex, resistance to serum destabilization, and improved intracellular dissociation kinetics.(23) Different hydrophobic groups have been introduced into the chitosan backbone, such as deoxycholic acid,(24) palmitic acid,(25) stearic acid,(26) and $\alpha$ tocopherol,(27) among others.

Specifically in this work, we have synthesized a stearic acid modified quaternary chitosan, and investigated it regarding complexation with an SSO, physicochemical characteristics of the nanocomplexes, and stability under different physiological conditions, as well as cellular delivery concerning the efficiency and mechanisms of intracellular uptake. We, for the first time, both show the successful use of a chitosan based vector for the delivery of SSOs in vitro, under serum conditions, and report on the influence of a hydrophobic modification in this process.

\section{Materials and Methods}

\section{Trimethylchitosan purification and modification}

Trimethylated chitosan (TMC) derived from ultrapure chitosan produced from Agaricus bisporus mushrooms, under cGMP, was provided by Kytozyme (Belgium) (reported average viscosimetric molecular weight obtained from starting chitosan $40000, \mathrm{~N}$-acetyl-glucosamine content $8.45 \mathrm{~mol} \%$, and methylation content ( $\mathrm{O}$-substituted and $\mathrm{N}$-trisubstituted) $28.82 \mathrm{~mol} \%$ ). Prior to use, the polymer was purified by filtration and dialysis. Briefly, TMC was dissolved in $5 \mathrm{mM} \mathrm{HCl}$ solution at a final concentration of $0.5 \%(\mathrm{w} / \mathrm{v})$, filtered through a sintered glass filter (pore size 100-160 $\mu \mathrm{m}$, Duncan), dialyzed for 3 days against deionized (DI) water and collected after freeze-drying $\left(-80{ }^{\circ} \mathrm{C} / 72 \mathrm{~h}\right.$, Labconco). The purified polymer was then further characterized. Polymer molecular weight was determined by gel permeation chromatography (GPC), with measurements performed in $0.33 \mathrm{M}$ $\mathrm{NaCH}_{3} \mathrm{COOH} / 0.28 \mathrm{M} \mathrm{CH}_{3} \mathrm{COOH}$ eluent at a flow rate of $1 \mathrm{~mL} \cdot \mathrm{min}-1$. The average number molecular weight $(\mathrm{Mn})$ of the purified material was found to be $43.3 \pm 5.5 \mathrm{kDa}$. The polydispersity $(\mathrm{Mw} / \mathrm{Mn})$ was

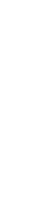


found to be $2.4 \pm 0.3$. The degree of acetylation (DA) was determined by Fourier-Transform Infrared Spectroscopy (FTIR) according to Brugnerotto et al.,(28) and found to be $11.1 \pm 0.9 \%$. The quaternization degree (DQ) was assessed by $1 \mathrm{H}$-Nuclear Magnetic Resonance Spectroscopy $(1 \mathrm{H}$ NMR). Polymer samples were dissolved in $\mathrm{D}_{2} \mathrm{O}(2 \mathrm{mg} \cdot \mathrm{mL}-1)$ at $60^{\circ} \mathrm{C}$ overnight and further analyzed in a digital spectrometer (Bruker Avance III 400) at room temperature with a total of 200 scans. The calculation of \% DQ was performed according to Mourya et al.(29) and was found to be $30.1 \pm 4.6 \%$. Endotoxin levels of the purified polymer extracts were assessed using the Limulus Amebocyte Lysate Assay (QCL-1000, Cambrex), following the manufacturer instructions. Endotoxin levels were found to be $<0.1 \mathrm{EU} \mathrm{mL}-1$, respecting the US Department of Health and Human Services guidelines for implantable devices.(30)

The purified TMC polymer was functionalized with stearic acid (SA) moieties by amidation of glucosamine residues through the reaction of TMC with $\mathrm{N}$-succinimidyl stearate (Santa Cruz BioTechnologies, USA). In brief, $50 \mathrm{mg}$ of purified and predried $\left(60^{\circ} \mathrm{C}, 24 \mathrm{~h}\right.$ in a vacuum oven) TMC was solubilized in $0.1 \mathrm{M} \mathrm{MES}$ buffer $\mathrm{pH} 6.5(6.2 \mathrm{~mL})$ for $24 \mathrm{~h}$ with stirring. It was then transferred to a round-bottom flask with a reflux system at $50{ }^{\circ} \mathrm{C}$ after which $9.4 \mathrm{~mL}$ of methanol was added dropwise. The mixture was vigorously stirred for $2 \mathrm{~h}$ and a solution of $\mathrm{N}$-succinimidyl stearate in methanol $(0.26 \% \mathrm{w} / \mathrm{v})$ was then added dropwise to the TMC solution at $50^{\circ} \mathrm{C}$ and left to react for 24 h. Subsequently the solution was dialyzed (Spectra/Por 3 Dialysis Membrane, MWCO 3.5 kDa) against distilled water for $72 \mathrm{~h}$, lyophilized and subsequently washed with ethanol to remove unreacted stearic acid. The resulting TMC-SA was then dispersed in DI water and lyophilized for $72 \mathrm{~h}$. NMR spectra were recorded in a Bruker Avance III $400 \mathrm{MHz}$ spectrometer in $2.7 \% \mathrm{DCl}$ in $\mathrm{D}_{2} \mathrm{O}: \mathrm{CD}_{3} \mathrm{OD}(1: 1)$ at $323 \mathrm{~K}$. Chemical shifts are reported in ppm ( $\delta$ units) downfield from internal 3-(trimethylsilyl)propionic acid-d4.

The degree of substitution (DS) for TMC-SA was determined by integration of the appropriate signals (stearic acid methyl group and TMC acetyl groups) in the $1 \mathrm{H}$ NMR spectrum, and calculated by the following equation.

$D S(\%)=\left[\frac{\int \mathrm{CH}_{3}(0.8 \text { to } 0.9 \mathrm{ppm})_{\text {Sternitikid }}}{\int \mathrm{CH}_{3}(2 \text { to } 2.2 \mathrm{ppm})_{\text {TMC }}}\right] \times 0.111 \times 100$

\section{Oligonucleotides}

The splice switching oligonucleotide $\mathrm{SSO}_{705}$, an 18nt single-stranded fully phosphorothioated 2'-Omethyl RNA oligonucleotide ( 5 '- CCU CUU ACC UCA GUU ACA), is directed against an aberrant splice site that is created in $\beta$-thalassemic patients by a $T$ to $G$ mutation at nucleotide 705 of intron 2 of the human $\beta$-globin gene.(31) A Cy5-labeled $\mathrm{SSO}_{705}$ was additionally obtained for flow cytometry and fluorescence microscopy analysis. Oligonucleotides were obtained from Integrated DNA Technologies, USA.

\section{Polymer:oligonucleotide complex formation}

Predried polymers $\left(60^{\circ} \mathrm{C}, 24 \mathrm{~h}\right.$ in vacuum oven) were dissolved in a two-step procedure in order to obtain a $0.3 \%(\mathrm{w} / \mathrm{v})$ polymer solution. Initially, $1 / 2$ volume of $5 \mathrm{mM} \mathrm{HCl}$ was added to the polymer and incubated overnight at $37^{\circ} \mathrm{C}$ with no agitation. Then, $1 / 2$ volume of $40 \mathrm{mM} \mathrm{HEPES,} 10 \%(\mathrm{w} / \mathrm{v})$ glucose,

西 
$\mathrm{pH} 7.3$ was added to the previous solution. The solution was incubated for additional $8 \mathrm{~h}$ with agitation to complete the dissolution procedure. Subsequently, the polymer solution was filtered through a $0.22 \mu \mathrm{m}$ pore sized filter unit (Millex-GP, Millipore) and polymer concentration was corrected by calculating the recovery of the polymer using Cibacron Brilliant Red (CBR) (SigmaAldrich). The method is based on the interactions of the anionic CBR molecule with the cationic polymer (electrostatic interactions) giving a shift of its absorbance at $575 \mathrm{~nm}$ when in a complexed state.(32) In brief, polymer solutions were incubated with CBR $\left(0.75 \mu \mathrm{g} \cdot \mathrm{mL}^{-1}, \mathrm{pH}\right.$ 9) and left to react for $20 \mathrm{~min}$ at room temperature (RT) under mild agitation (80o rpm, Thermomixer). Absorbance (575 $\mathrm{nm}$ ) was recorded using a multimode microplate reader (SynergiMX, BioTek, USA), against a standard curve ( 0.2 to $1.2 \mathrm{mg} \cdot \mathrm{mL}^{-1}$ ) made with an unfiltered polymer sample of known concentration.

Complexation was achieved by mixing equal volumes of polymer and oligonucleotide diluted in buffer (20 mM HEPES, $5 \%$ (w/v) glucose, pH 7.3) supplemented with a final concentration of $50 \mathrm{mM}$ of $\mathrm{Na}_{2} \mathrm{SO}_{4}$. Polymer and $\mathrm{ON}$ solutions were heated at $60{ }^{\circ} \mathrm{C}$ before mixing under vortex and the resulting mixture was further incubated at $60^{\circ} \mathrm{C}$ for 10 min under agitation (1000 rpm, Thermomixer). The complexes were allowed to stabilize for at least $30 \mathrm{~min}$ at RT before use. Complexes with different molar ratios of quaternized amine groups $(N)$ to oligonucleotide phosphate groups $(P)-N / P$ molar ratio-were prepared. Unless otherwise stated, a final concentration of $1.5 \mu \mathrm{M}$ of oligonucleotide was used during complex formation.

\section{Polyacrylamide gel retardation assays}

Complexes were prepared at different N/P ratios after which an aliquot corresponding to 6 pmol of $\mathrm{ON}$ was diluted in $\mathrm{H}_{2} \mathrm{O}$ and $6 x$ Loading buffer, and loaded in 4-20\% (w/v) polyacrylamide-TBE gels (Novex TBE Gels, Invitrogen). Gels were stained postrun with SYBRGold (Molecular Probes, Invitrogen) in 1X TBE solution. Gels were visualized in a GelDoc XR imaging system (BioRad) and analyzed using IMAGELAB software (BioRad).

For analysis of heparin-induced dissociation of complexes, these were prepared at N/P ratio 80 after which an aliquot was diluted in $1 x$ phosphate buffer saline containing different Heparin concentrations (previously diluted in $1 \times$ PBS also). Complexes and heparin were then incubated for 2 $\mathrm{h}$ at $37^{\circ} \mathrm{C}$. After incubation complexes (corresponding to $6 \mathrm{pmol} \mathrm{ON}$ ) were loaded in $4-20 \%$ polyacrylamide-TBE gels and stained postrun with SYBRGold (Molecular Probes, Invitrogen).

\section{Dynamic light scattering, zetapotential and cryo-scanning electron microscopy}

Hydrodynamic mean diameter of nanocomplexes was determined by dynamic light scattering (DLS) studies using a Zetasizer Nano ZS (Malvern, UK). Complexes were prepared at different N/P ratios and analyzed either undiluted or, in the case of stability studies, further diluted 3 -fold in $1 \times$ PBS or DMEM with $10 \%$ fetal bovine serum (FBS).

Zetapotential of the free polymer or nanocomplexes, diluted 3 -fold in complexation buffer or DMEM with $10 \%$ FBS, was measured using the above instrument.

SEM analysis of the nanocomplexes was performed using a High-Resolution Scanning Electron Microscope and CryoSEM experimental facilities (JEOL JSM 6301F/Gatan Alto 2500). The samples 
were rapidly cooled (plunging it into subcooled nitrogen-slush nitrogen) and transferred under vacuum to the cold stage of the preparation chamber. The samples were then fractured, sublimated ("etched") for $120 \mathrm{~s}$, at $-90^{\circ} \mathrm{C}$, and coated with Au/Pd by sputtering for $40 \mathrm{~S}$, after which they were transferred to the SEM chamber. Analysis was performed at $-150^{\circ} \mathrm{C}$.

\section{Cell transfections}

HeLa/Luc705 cells (a kind gift from Prof. R. Kole and Prof. R. Juliano) stably transfected with pLuc705 plasmid where the luciferase sequence, used as a reporter gene, is interrupted by a mutated $\beta$-globin intron,(31) were grown at $37^{\circ} \mathrm{C}, 5 \% \mathrm{CO}_{2}$ in DMEM with Glutamax (Invitrogen) supplemented with $10 \%$ FBS (Gibco) and $35 \mu \mathrm{g} / \mathrm{mL}$ gentamycin (Sigma).

Cells were seeded $\left(6 \times 10^{4}\right.$ cells in $\left.500 \mu \mathrm{L}\right)$ into 24 -well plates, $24 \mathrm{~h}$ prior to transfections (resulting in approximately $75 \%$ confluency at the day of transfection). TMCs/ON nanocomplexes were prepared at different N/P ratios as previously described. Cell medium was replaced prior to transfections with fresh medium with no antibiotics and nanocomplexes added corresponding to maximum $20 \%$ of the final volume ( $60 \mu \mathrm{L}$ of nanocomplexes in $300 \mu \mathrm{L}$ final volume). Lipofectamine 2000 (L2k) (Invitrogen) was used as a control according to the manufacturers instructions. Complexes were incubated with cells for different time periods $(8,24,48$, and $72 \mathrm{~h}$ ). For periods longer than $24 \mathrm{~h}$ an additional $300 \mu \mathrm{L}$ of medium with no antibiotics was added. At the end of the incubation periods, cells were lysed using $300 \mu \mathrm{L} 0.15 \%(\mathrm{v} / \mathrm{v})$ Triton X-100 in HEPES-buffered Krebs Ringer (HKR) buffer for 5 min on ice after which they were frozen at $-80^{\circ} \mathrm{C}$. Luciferase activity was measured using Promega's luciferase assay system with luminescence readings on a multimode microplate reader (SynergiMX, Biotech) that were normalized to protein content (BCA assay, ThermoFisher/Pierce, USA). For RT-PCR, cells were incubated with chitosanase (CALBIOCHEM) $\left(6.5 \mathrm{mU}\right.$ per ug chitosan) for $1.5 \mathrm{~h}$ at $37^{\circ} \mathrm{C}$, prior to proceeding with total RNA isolation. RNA isolation was performed using mirVana miRNA isolation kit (AMBION). A total of $10 \mathrm{ng}$ of RNA was then used in each reaction (total volume per reaction was $25 \mu \mathrm{L}$ ) with the ONE STEP RT-PCR kit (QIAGEN) and following the manufacturers protocol. The primers used were: Fwd-TTGATATGTGGATTTCGAGTCGTC; TGTCAATCAGAGTGCTTTTGGCG. The program for the RT-PCR was as follows: $\left(55^{\circ} \mathrm{C}, 35 \mathrm{~min}+95\right.$ $\left.{ }^{\circ} \mathrm{C}, 15 \mathrm{~min}\right) \times 1 \mathrm{cycle}+\left(94^{\circ} \mathrm{C}, 30 \mathrm{~s}+55^{\circ} \mathrm{C}, 30 \mathrm{~s}+72{ }^{\circ} \mathrm{C}, 30 \mathrm{~s}\right) \times 29-30$ cycles $+72{ }^{\circ} \mathrm{C}, 10 \mathrm{~min}$ final extension. The PCR products were analyzed in a $2 \%$ agarose gel in $1 \times$ TAE buffer previously stained with SYBRGold (Invitrogen, Molecular Probes). Gel images were captured with a GELDOC system (BioRad) with the ImageLab software (BioRad).

In experiments with chloroquine (CQ), a final concentration of $100 \mu \mathrm{M}$ was used. $\mathrm{CQ}$ was added to cells $20 \mathrm{~h}$ post-transfection to promote endosomal escape of endosomal-retained complexes. Four hours after addition of $\mathrm{CQ}$ to cells, cell medium was replaced with fresh medium in order to avoid the toxic effects of $\mathrm{CQ}$, and incubation proceeded for further $24 \mathrm{~h}$ (total incubation time of $48 \mathrm{~h}$ posttransfection).

\section{Cell metabolic activity assay}

HeLa/Luc70 5 were seeded in 24 -well plates ( $4 \times 10^{4}$ cells), $24 \mathrm{~h}$ prior to transfection in order to have $50 \%$ confluency. Nanocomplexes prepared as described previously and L2k based complexes were incubated with cells for $24 \mathrm{~h}$. Medium was then replaced with $300 \mu \mathrm{L}$ of fresh complete medium containing $10 \%(\mathrm{v} / \mathrm{v})$ resazurin (from an initial stock of $0.1 \mathrm{mg} / \mathrm{mL}$ in PBS, Sigma). Cells were 
incubated for $1.5 \mathrm{~h}$ at $37^{\circ} \mathrm{C}$ after which $200 \mu \mathrm{L}$ of the medium was transferred to a black 96 -well plate (Brand Plates, Brand GMBH, Germany) and fluorescence was measured ( $k_{\mathrm{exc}}=530 \mathrm{~nm}, k_{\mathrm{em}}=590 \mathrm{~nm}$, SynergiMX, Biotech). To determine background fluorescence levels, nontreated cells were incubated for 45 min with $0.5 \%(\mathrm{v} / \mathrm{v})$ Triton X-100, to achieve total cell lysis, before resazurin incubation. Results are expressed as percentage of metabolic activity of treated cells relative to untreated cells.

\section{Internalization assays}

For flow cytometry analysis, cells were seeded $\left(6 \times 10^{4}\right.$ cells) $24 \mathrm{~h}$ prior to transfections in 24 -well plates. Cells were incubated with nanocomplexes as previously described, for 4 or $24 \mathrm{~h}$. After the mentioned time periods, cells were washed twice with $1 \times$ PBS, trypsinized, again washed twice with PBS and finally resuspended in PBS containing $2 \%(v / v)$ FBS. Cells were run on a FACSCalibur flow cytometer (BD Biosciences) and data analyzed using FlowJo software (version 10, FLOWJO, LLC).

For laser scanning confocal microscopy, HeLa/Luc705 cells were seeded on an 8-well IBIDI $\mu$-slide, IBItreat (IBIDI, Germany) the day before transfection. Complexes of TMC-SA/Cy5-ON at N/P 80 and Tetramethylrhodamine-Dextran (70 kDa) (Molecular Probes, Life Technology, USA) were coincubated with cells for 4 or $24 \mathrm{~h}$. After washing with PBS, cells were counterstained with Hoechst 33342 in $1 \times$ PBS for nuclei identification (Molecular Probes, Life Technology, USA) for 5 min and again extensively washed with PBS. Finally OPTI-mem (no phenol red) (Invitrogen, Life Technologies, USA) was added to the wells. Live cell imaging was performed on a Leica $\mathrm{SP}_{2}$ AOBS SE (Leica Microsystems, Germany) inverted microscope equipped with a Plan-Apochromat $63 \times / 1.4$ oil objective with a 0.8 aperture. Images were acquired using Z-stack mode with 30 slices corresponding to steps of $0.204 \mu \mathrm{m}$, and a pinhole of $1 \mathrm{AU}$ in all channels. ImageJ software, (version 2.0.0-rc15/1.49m), was used for analysis and preparation of images.

\section{Statistical analysis}

Statistical analysis was performed using GraphPad Prism 5.o. One-way or two-way ANOVA was used accordingly and as mentioned in each figure.

\section{Results}

\section{Characterization of trimethylchitosan functionalized with hydrophobic moieties}

Purified TMC (Mn = 43.3 kDa) was functionalized with a fatty acid chain, stearic acid (SA), by reaction between free primary amines from its glucosamine residues and succinimidyl-stearate (Scheme 1 ). The grafting of the stearate chains was confirmed by $1 \mathrm{H}$ NMR due to the appearance of the signals corresponding to the stearic acid protons: a triplet at $0.8 \mathrm{ppm}$ (methyl group), a sharp singlet at 1.30 ppm (methylene protons), and a multiplet at 1.44-1.51 ppm ( $\alpha$-methylene protons) (Figure 1). The average degree of substitution (DS), defined as the number of SA groups per 100 sugar units of TMC, was calculated by comparing the ratio of methyl protons of stearic acid $(\delta=0.8-0.9)$ to acetyl protons of the $\mathrm{N}$-acetyl glucosamine residue of TMC $(\delta=2.0-2.1 \mathrm{ppm}$ ) and was found to be $2.1 \pm 0.6 \%$ (mol $\%)$.

\section{TMC and TMC-SA complexation with SSOs}

(1) 
The interaction strength between the polymer and oligonucleotide was initially assessed by a polyacrylamide gel retardation assay (Figure 2). Polyacrylamide gel electrophoresis (PAGE) was chosen, as it gives a higher resolution for ssONs detection and separation, in comparison to agarose gels. Under the gel running conditions, it was possible to verify an almost complete retention of the SSO from N/P ratio 4 for TMC and from N/P ratio 3 for TMC-SA.

Complexes between the polymers and SSO were also characterized using DLS (Figure 3). Both TMC and TMC-SA based complexes show a rather narrow particle size distribution between the different $\mathrm{N} / \mathrm{P}$ ratios (ranging from 115 to $180 \mathrm{~nm}$ in average diameter). Particle size decreases with increasing N/P ratios until N/P 20-40, above which sizes start to slightly increase again, although no statistically significant differences have been found.

Significant differences between the complexes of both polymers are observed for the polydispersity index $(\mathrm{Pdl})$, which is maintained rather constant at values $\leq 0.3$ for TMC-SA, whereas for TMC this rises above 0.5 for $N / P \geq 40$.

\section{Transfection efficiency of the nanocomplexes}

As the average diameters found for the complexes showed sizes in line with transfection competent particles, they were tested in a splice correction model system cell line, HeLa/Luc705.(31)

Hence, transfection efficiency of nanocomplexes was tested at different N/P ratios for both the unmodified TMC and TMC-SA in serum containing cell culture medium (Figure $4 \mathrm{~A}$ ). A clear increased efficiency for complexes of TMC-SA was seen, especially at higher N/P ratios (above N/P 40). The hydrophobic modification was indeed crucial to achieve significant levels of transfection efficiency, as the unmodified TMC clearly lacked any appreciable capacity to deliver these oligonucleotides. When compared to a known commercial transfection reagent (Lipofectamine 2000) used as a gold standard for this type of in vitro transfections, the efficiency of TMC-SA was just approximately 2fold less under the conditions tested. As an additional control, free $\mathrm{SSO}_{70} 5$ (without any transfection reagents) was incubated with the cells at $1 \mu \mathrm{M}$ final concentration for $48 \mathrm{~h}$, showing no increase of luciferase activity even at this high concentration (Figure $4 \mathrm{~A}$, graphical insert). To confirm correction at the level of RNA, cells were transfected with the nanocomplexes at different SSO concentrations, at N/P ratio 80 (shown to be of highest efficiency), and total RNA was isolated and subjected to RTPCR (Figure $4 \mathrm{~B}$ ). Results confirmed the correction of the aberrant Luciferase RNA after transfection.

Transfection activity was also evaluated at different time points (Figure ${ }_{4} \mathrm{C}$ ) with complexes formed at N/P ratio 80 . Transfection, as measured by the increase in luciferase activity over untreated cells, increased up to the $48 \mathrm{~h}$ time point.

Cytocompatibility of the nanocomplexes was evaluated by measuring cellular metabolic activity. When incubating the nanocomplexes with cells for a period of $24 \mathrm{~h}$, a slight decrease of the cytocompatibility was observed as cell metabolic activity was reduced by around $30 \%$, for N/P ratios $20-80$ and around $45 \%$ for N/P ratio 160 . In comparison, L2k control used at the same SSO dose gave a reduction of metabolic activity of $80 \%$ (Figure $4 \mathrm{D}$ ).

\section{Colloidal stability and zeta potential of transfection competent nanocomplexes}

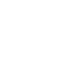


To further unravel the contributions of hydrophobically modified TMC-SA in the formation of transfection competent complexes, size and zeta potential were measured after incubation in serum containing medium (Figure 5 and Table 1). Nanocomplexes were thus analyzed by DLS before and after dilution with $1 \times$ PBS or DMEM $+10 \%$ FBS. The intensity versus size profile obtained clearly indicates that incubation of the TMC particles (Figure $5 \mathrm{~A}$ ) with serum containing medium induced an increase of the maximum intensity peak width and a shift toward increased sizes, but also the appearance of small sized peaks distinct from the serum protein background. For TMC-SA nanocomplexes (Figure ${ }_{5} \mathrm{~B}$ ) no significant difference is seen between the size profiles.

Zeta potential of the nanocomplexes was also measured (Table 1). Both types of particles displayed positive values (from +7.2 to $8.9 \mathrm{mV}$ ), which significantly decrease when incubation with serum containing medium is performed. This likely reflects the tendency for proteins to adsorb to the surface of the nanoparticles, thereby shielding part of the surface charge. For comparison, the zeta potential of the free polymers in HEPES buffer solution is shown, revealing a much higher zeta potential (from +18.0 to $19.6 \mathrm{mV}$ ). The difference from the formed nanocomplexes reflects the influence of the SSOs and sodium sulfate used for particle formation.

The morphology of transfection competent nanocomplexes was assessed using cryo-SEM, where it was observed that particles were mostly regular spherical shaped, with sizes, in line with the DLS measurements (Supporting Information Figure 1).

Another important parameter for the cellular activity of the transfection complexes relates to their capacity to release the nucleic acids once inside the cell. Thus, to test the reversibility of complex formation, we incubated the complexes with heparin, a model polyanion commonly used to test destabilization and release of nucleic acids from polyplexes. $(33,34)$ The incubation was performed at $37^{\circ} \mathrm{C}$ and at physiological salt and $\mathrm{pH}$ conditions (Figure 6). Both TMC-SA and TMC-based complexes, formed at N/P ratio 80 , were tested, and both showed extensive release of SSOs when challenged with heparin.

Figure 6. Heparin dissociation assay. Complexes formed between TMC and TMC-SA with SSOs, using a N/P ratio of 80 , were incubated with increasing concentrations of heparin at $37^{\circ} \mathrm{C}$ in physiological salt and $\mathrm{pH}$ conditions for $2 \mathrm{~h}$. Samples were then run using PAGE to verify the extent of dissociated SSOs from the complexes. In the SSO lane the same amount of free oligo as occurring in the nanocomplexes was loaded.

\section{Cellular association and intracellular distribution of transfection competent nanocomplexes}

The correlation between the functional effect of TMC-based SSO complexes, as evaluated by the luciferase activity assays, and the degree of cellular association of the complexes was then examined. Nanocomplexes formed with a fluorescently labeled SSO were used in flow cytometry experiments (Figure 7). Cells were analyzed after 4 or $24 \mathrm{~h}$ incubation with the nanocomplexes. Of notice is the fact that this method can only give information on the total cellular association without discriminating between membrane-associated or internalized SSO-complexes. The results showed, for both time periods, a complete shift in the population of cells associated with Cy5 fluorescence. Cells treated with TMC-SA-based nanoparticles show, for both time points, a higher fluorescence intensity in comparison to the ones treated with TMC nanoparticles and control free ON, but below

.


L2k. As expected, the highest shift in fluorescence intensity is seen at $24 \mathrm{~h}$ for all the conditions; however, comparing the fluorescence intensity of free $\mathrm{ON}$ with both polymer-based nanoparticles and $L 2 k$ one can distinguish two populations of cells, one with fluorescence intensities similar to free $\mathrm{ON}$ and another with higher fluorescence intensity (highlighted area in graph, Figure 7).

To more closely examine the uptake and intracellular distribution of the transfection competent

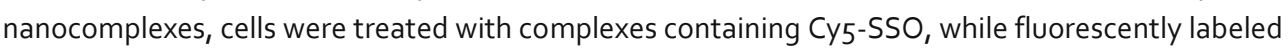
$70 \mathrm{kDa}$-Dextran was concomitantly used during the incubation periods to assess for fluid phase endocytosis (Figure 8). Live cell imaging was used, as fixation is often associated with uptake and intracellular distribution artifacts of positively charged molecules.(35) As chitosan is known to present slow transfection kinetics, imaging was done at $4 \mathrm{~h}$ (Supporting Information Figure 2) and 24 h (Figure 8) time points (post-transfection).(36) At both time points, clear punctuated structures are visible, indicating preferential localization in vesicles, with the presence of some colocalization spots between dextran and $\mathrm{Cy}_{5}$-SSO showing trafficking through the endosome/lysosome pathway. In addition, at the $24 \mathrm{~h}$ time point, a diffuse nuclear fluorescence is clearly visible, indicating that the $\mathrm{Cy}_{5}$-SSO has been released from the endo/lysosomes and dissociated from polymer (Supporting Information Figure 3). Interestingly, bright fluorescence was also visible at the cell membrane, at both time points, indicating accumulation or aggregation of $\mathrm{Cy}_{5}-\mathrm{SSO}$ containing complexes for an extended period of time.

In addition, at this time point, the nucleus presents a diffuse red fluorescence indicating accumulation of $\mathrm{Cy}_{5}$-SSO that escaped from the vesicles and dissociated from the polymer.

Finally, to further confirm the presence and evaluate the extent of localization of nanocomplexes inside lysosomes, transfection activity was evaluated after $\mathrm{CO}$ was added to cells incubated with nanocomplexes. Chloroquine is a known disruptor of endo-/lysosomal vesicles acting through a buffering mechanism that increases vesicle osmotic pressure.(37) When added $24 \mathrm{~h}$ posttransfection, a 100\% increase in efficiency ( 2 -fold) was appreciated (with the effect paralleling that of L2k) (Figure 9).

\section{Discussion}

Splice switching oligonucleotide-based therapies have harnessed a great deal of interest due to the increasing number of human diseases known to occur because of defective splicing. Nevertheless, SSO delivery and efficacy still need to be improved for this strategy to fulfill its potential.(38) Chitosan, a natural biodegradable and biocompatible polysaccharide-based polymer, is seen as a very promising nucleic acids delivery system aiming at future clinical applications.(16) However, to the best of our knowledge, chitosan has never been explored as a vector for the delivery of SSOs. In this work, we address the capabilities of a chitosan-based vector to form stable nanocomplexes with an SSO and assess its delivery efficiency. SSOs belong to the category of single-stranded oligonucleotide therapeutics and are inherently different, regarding their physicochemical properties, from both pDNA and siRNA. As short single-stranded oligonucleotides (usually around 14-25 nucleotides in length), they present low negative charge density and have a slight apolar character, as the nucleobases are not participating in the formation of hydrogens bonds, as in the double-helix structure. Hence, the favorable polymer properties and complexation conditions for SSO delivery are intrinsically different from the ones found for pDNA or siRNA. $(39,40)$ 
Considering the properties of SSOs, here we explored the use of a trimethylchitosan (TMC) conjugated with hydrophobic 18-carbon acyl chains (stearate chain), as an SSO delivery vector.

Upon comparing the association strength of the stearylated trimethylchitosan (TMC-SA) and the unmodified TMC with an SSO, no major differences were seen as both associated strongly from N/P ratio $3-4$, respectively with $>90 \%$ of SSO bound to the polymers. Particles were run in a polyacrilamide gel (at $\mathrm{pH}$ 8), and under these conditions no apparent effect of the extra hydrophobicity imparted by the stearyl units was visible. Of notice is the fact that complex formation was performed using a coacervation method aided by sodium sulfate. Sodium sulfate is known to participate in chitosan particle formation through a mechanism of ionic cross-linking but also acting as a desolvating agent that, by aiding removal of water molecules from polymer chains, increases intramolecular hydrophobic interactions. $(41,42)$ Thus, this extra stabilization conferred by sodium sulfate could explain the apparent similarities between TMC-SA and TMC in the gel retardation assay.

In terms of particle average sizes, no significant differences were found between polymers, except for the lowest ratios tested (N/P ratios 5 and 10), where TMC-SA formed slightly smaller sized particles. The result for the lower N/P ratios can be explained by the increased tendency for selfassociation (aggregation) of hydrophobically modified chitosan. $(24,43)$ On the other hand, a big difference was observed regarding Pdl values. From N/P ratio 20 onward, whereas TMC-SA maintained a constant Pdl around 0.3 , unmodified TMC presents a very heterogeneous population with Pdls above 0.5 . This effect can be accounted for by the different aggregation behavior between unmodified TMC and hydrophobic TMC. While the aggregation behavior of chitosan and hydrophobic chitosan derivatives have been reported, the diversity of characteristics of the polymers being used makes it difficult to draw precise conclusions through comparison of data.(44) Nevertheless, the presence of hydrophobic groups imparts a self-association behavior onto chitosan with formation of stronger intermolecular interactions and stable core-shell structures, which contributes to the formation of stable complexes in a wider range of polymer concentrations (in this case, defined by the N/P ratio). $(44,45)$

As complexes were displaying properties favorable for cell delivery, the transfection capacity of both polymers was screened at different N/P ratios. Strikingly, the unmodified TMC showed an almost complete lack of activity for all N/P ratios tested, while use of the modified TMC-SA resulted in efficient transfection, with N/P ratio 80 displaying the highest activity (Figure $4 \mathrm{~A}$ ). Under the conditions tested, the maximum transfection efficiency achieved corresponded to $50 \%$ of the levels reached by the commercial in vitro transfection reagent L2k. Additionally, the activity of the SSO delivered by the TMC-SA nanocomplexes was verified at the level of RNA, where a significant degree of correction was observed; albeit, the highest concentration tested was necessary for this effect (Figure $4 \mathrm{~B}$ ). This confirmed the potential of the hydrophobic modification in significantly increasing the single-stranded oligonucleotide transfection potential of chitosan. Of notice is the fact that a rather high N/P ratio was needed to achieve good transfection activity. Despite some studies reporting the use of low N/P ratios for complexation and transfection of ssONs, a clear relation between N/P ratio and transfection activity has not been established. $(17,46,47)$

Possibly, under our study conditions, the high N/P ratios used to achieve good transfection efficiency relate to the stabilization of the nanocomplex, but also the influence of the excess of free polycation on promoting lysosomal release of polyplexes has recently been suggested.(48) Use of high N/P 
ratios is comparable to what has been described for siRNA, as one can find very broad N/P ratios being used ranging from low (N/P 5-10)(49) to very high (N/P 150).(50)

Cell viability was evaluated by measuring metabolic activity after incubation with the nanocomplexes at different N/P ratios. TMC-SA nanocomplexes proved to be much less cytotoxic than L2k. While a decrease of $30 \%$ in metabolic activity was observed, this could be a consequence of the high N/P ratios used under these conditions (Figure ${ }_{4} \mathrm{D}$ ); still, this level of toxicity needs to be taken into consideration and improved when developing this system further for in vivo applications.

To further characterize the transfection activity of TMC-SA/SSO nanocomplexes, kinetic studies were performed. A late onset of activity was verified as maximum luciferase induction (fold increase) occurred at the $48 \mathrm{~h}$ time point (Figure ${ }_{4} \mathrm{C}$ ), in line with the slow transit kinetics previously described for chitosan-based vector systems.(36) These kinetics have been linked with a long residence time of chitosan-based systems in lysosomes. $(36,51)$ In agreement with this hypothesis, a $100 \%$ increase in transfection activity (2-fold increase) was observed when challenging cells with $\mathrm{CO}, 24 \mathrm{~h}$ postincubation with nanocomplexes (Figure g). This strong increase implies that a significant protection of the oligonucleotide from degradation is achieved when trafficking through the endolysosomal pathway. Additionally, it indicates that also in the case of our chitosan-SSO system there would be an added benefit of improving the buffering capacity of the polymer, as previously attempted, including by us. $(36,52,53)$ Nevertheless, stable chitosan-DNA polyplexes may benefit from some degree of chitosan degradation in lysosomes in order to weaken the polyplex and facilitate subsequent cytoplasmic dissociation of the nucleic acid. $(36,54)$

To elucidate the mechanisms responsible for the difference of activity between both polymers, the colloidal stability of nanocomplexes challenged with serum containing medium was evaluated (Figure 5). Binding of negatively charged proteins and other components found in serum can lead to both aggregation and disassembly of polyplexes in the extracellular medium, and determination of this effect is crucial for predicting the biological activity of nanocomplexes. (55-57) Indeed, results showed a different behavior of unmodified TMC and TMC-SA even in the presence of diluted serum containing medium. Whereas TMC-SA based nanocomplexes showed a very good colloidal stability, verified by the similar size distribution profile of the particles incubated with or without serum, unmodified TMC displayed both a broadening of its size distribution profile, indicating an aggregation behavior, and the appearance of smaller sized peaks (different from the obtained when only measuring serum containing medium-blank), indicative of disassembly of the nanocomplex into smaller components. Thus, this distinct behavior is well correlated with the transfection activity differences obtained between both polymers. Surface charge of the complexes (Table 1 ) was found at the same level (between +5 to $10 \mathrm{mV}$ ) with other reports where complexation is performed with the aid of sodium sulfate as an electrostatic cross-linker. $(41,58)$ Zeta potential is then mostly influenced by the close interaction of sulfate ions with positively charged amine groups from the macromolecular chitosan coils. (42) After incubation with serum containing medium, a decrease in the zeta potential for both TMC polymers was observed. This indicated that negatively charged proteins are in close interaction with the polyplexes, with unmodified TMC being the most affected by this process, as described above. While stability of the polyplexes is an important feature, especially when in the extracellular milieu, it is important that, during the intracellular stages, especially when in the cell cytoplasm, the complexes are reversible, meaning that the SSOs are able to dissociate from the polyplexes in order to be active. To this end, we investigated the behavior of the polyplexes when incubated with heparin, a model polyanion. $(33,34)$ Additionally, the incubations 
were performed at intracellular salt and $\mathrm{pH}$ conditions to more closely resemble intracellular conditions. Competition by increasing concentrations of the polyanion showed the possibility of dissociation of the SSO for the higher end of concentrations tested. Together with the results from the colloidal stability when in the presence of serum, the dissociation of the polyplexes after heparin incubation demonstrated that at the N/P ratio of 80 (used for successful transfection) there can still be a good equilibrium in terms of extracellular stability and intracellular release of the SSOs.

Nanocomplexes formed by both polymers were also evaluated regarding their cellular association behavior and at two different time-points ( 4 and $24 \mathrm{~h}$ ) post-transfection. Flow cytometry results revealed, independently of the evaluation time point, a complete shift in the population of cells associated with $\mathrm{Cy}_{5}$ fluorescence for all the conditions tested (Figure 7). The fluorescence intensity observed for cells treated with free SSO can be explained by the fact that being a phosphorothioate (PS) modified oligonucleotide it has a tendency to associate with cell membranes especially when in the presence of serum proteins. This is a well-known effect responsible for free PS-modified oligonucleotide uptake in vitro and in vivo,(59) albeit being mainly a nonproductive uptake, as most of the material accumulates in endosomes/lysosomes without cytoplasmic release.(60) Comparing the fluorescence intensities given by free SSO versus polymers and L2k, one could distinguish and categorize two populations of cells with low and high fluorescence intensities (Figure 7). This population of cells with high FL intensity could refer to cells containing free Cy5-SSO in the cytosol/nucleus after disassembly from cationic polymer and escape from endo-/lysosomes (i.e., cells with efficient transfection). Supporting this is the fact that $\mathrm{Cy}_{5}$ dyes are known to self-quench when in close proximity due to resonance energy transfer, (61) which can occur when the oligonucleotide is in a complex with a polymer or lipid $(62,63)$ (the complexed state would be more likely during vesicle transport but also possible to exist in the cytosol). The same fluorescence quenching effect could occur with free ON, as its PS backbone can promote unspecific interactions with serum proteins (especially albumin), effectively leading to the creation of low affinity ON-protein complexes, which can be uptaken by cells. $(64,65)$ Indeed, all cell uptake and transfection experiments were done in the presence of serum, thus promoting this effect. Having the above-mentioned in consideration, a higher level of association of the TMC-SA-based polyplexes with cells, in comparison to TMC polyplexes, was verified. Moreover, the fact that a higher fluorescence intensity was also observed indicates that the $\mathrm{Cy}_{5}$-SSO is being productively taken up by cells (i.e., trafficking through the endo/lysosomal pathway and being released in the cytoplasm, especially at the $24 \mathrm{~h}$ time point). It is expected that the lower FL intensity achieved with unmodified TMC is linked to its lower colloidal stability, as observed above. In addition, TMC polyplexes could suffer from disassembly in the cell culture medium, which can also explain the similar fluorescence intensity shift when compared to free SSO. Thus, these results support the transfection efficiency activity differences observed between both polymers.

To further complement the previous observations regarding transfection activity and flow cytometry experiments, live cell confocal imaging was performed using transfection competent TMC-SA polyplexes containing $\mathrm{Cy}_{5}$-SSO. At both time points tested, colocalization between polyplexes and dextran (a fluid phase endocytosis marker known to accumulate in lysosomes(66)) is apparent, but also the presence of separate fluorescence spots is visible (Figure 8 and Supporting Information Figure 2). A spread fluorescence coming from the cell membrane region at both time points, indicating some aggregation and extended residence time of the polyplexes at the membrane, was also visible. This membrane localization could be attributed to some degree of incorporation of the stearyl chains of the polymer in the hydrophobic cell membrane, leading to accumulation and

INSTITUTO DE INVESTIIGAÇÃO E INOVACCÃO EM SAÚDE UNIVERSIDADE DO PORTO

Rua Alfredo Allen, 208 4200-135 Porto Portugal +351220 408800 
possibly aggregation of the polyplexes at some membrane regions. This effect has been reported in cell surface modification studies with amphiphilic polymers and peptides. $(67,68)$ The lack of complete colocalization between dextran and TMC-SA can be explained by the multiple endocytic pathways that hydrophobic modified polymers can take during cell uptake, as previously reported for both modified PEI and chitosan. $(25,69)$ The extensive localization of TMC-SA polyplexes at the membrane could also affect the kinetics and routes of cell uptake.

Nevertheless, the polyplexes seem to traffic through the endolysosomal pathway and reside for extended periods of time in lysosomes, as colocalization between dextran and Cy5-SSO is observed at both the $4 \mathrm{~h}$ (which is sufficient for dextran to accumulate in lysosomes(66)) and $24 \mathrm{~h}$ time points. The increased transfection activity when adding CQ (Figure 9 ) also corroborates this hypothesis, as mentioned above.

After $24 \mathrm{~h}$, escape of SSO from the lysosomes is visible as a diffuse nuclear Cy 5 fluorescence is observed, indicating an accumulation in the nucleus (an effect attributed to phosphorothioate modified oligonucleotides $(70,71)$ ) (Supporting Information Figure 3). Also, the diffuse Cy5 fluorescence observed indicates that $\mathrm{SSO}$ disassemble from the polymer either during lysosomal escape or once in the cytoplasm, since a spotted pattern would be expected if the polyplexes were still assembled when in the nucleus, as previously observed.(36)

\section{Conclusions}

In this study we propose that providing chitosan with an amphiphilic character is essential for formation of stable complexes with single-stranded oligonucleotides under physiological conditions (including the presence of serum). Thus, by grafting stearyl units to trimethylchitosan, we achieved formation of nanocomplexes with a splice switching oligonucleotide (SSO) and demonstrate efficient delivery in a splice correction model. Our study provides perspectives on factors governing efficient complexation, cell uptake, and transfection by chitosan-oligonucleotide complexes, which have not been clearly addressed by the field so far.

In conclusion, new avenues were opened for the use of amphiphilic chitosan-based vector systems for the delivery of therapeutic oligonucleotides with splice switching activity.

\section{Acknowledgements}

This work was cofinanced by Fundação para a Ciência e a Tecnologia (FCT, Portugal) within projects HMSP-ICT/0020/2010 and PTDC/CTM-NAN/NAN/115124/2009. Additionally, P.M.D.M. acknowledges the support from the Marie Curie Actions of the European Community's 7th Framework Program (PIEF-GA-2011-300485); J.C.S. acknowledges the graduate fellowship from Conselho Nacional de Desenvolvimento Científico e Tecnológico (CNPq, Ministry of Science and Technology, Brazil); C.P.G. and V.L. acknowledge FCT for their scholarships (SFRH/BD/79930/2011 and SFRH/BPD/69110/2010). We thank M. Lázaro from the Bioimaging Center for Biomaterials and Regenerative Therapies (b.IMAGE) for help with confocal microscopy. 1 H NMR and Cryo-SEM were performed at the Centro de Materiais da Universidade do Porto (CEMUP).

Notes The authors declare no competing financial interest. 


\section{REFERENCES}

(1) Nilsen, T. W.; Graveley, B. R. Expansion of the eukaryoticproteome by alternative splicing.Nature2010,463(7280), 457-63

(2) Chen, J.; Weiss, W. A. Alternative splicing in cancer: implicationsfor biology and therapy.Oncogene2015,34(1), 1-14

(3) Tazi, J.; Bakkour, N.; Stamm, S. Alternative splicing and disease.Biochim. Biophys. Acta, Mol. Basis Dis. 2009,1792(1), 14-26

(4) Disterer, P.; Kryczka, A.; Liu, Y.; Badi, Y. E.; Wong, J. J.; Owen, J.S.; Khoo, B. Development of therapeutic splice-switching oligonucleo-tides.Hum. Gene Ther.2014, 25(7), 587-98

(5) Koo, T.; Wood, M. J. Clinical trials using antisenseoligonucleotides in duchenne muscular dystrophy.Hum. Gene Ther.2013,24(5), 479-88

(6) Passini, M. A.; Bu, J.; Richards, A. M.; Kinnecom, C.; Sardi, S. P.;Stanek, L. M.; Hua, Y.; Rigo, F.; Matson, J.; Hung, G.; Kaye, E. M.;Shihabuddin, L. S.; Krainer, A. R.; Bennett, C. F.; Cheng, S. H.Antisense oligonucleotides delivered to the mouse CNS amelioratesymptoms of severe spinal muscular atrophy.Sci. Transl. Med.2011,3(72), 72 ra18

(7) van de Vosse, E.; Verhard, E. M.; de Paus, R. A.; Platenburg, G. J.;van Deutekom, J. C.; Aartsma-Rus, A.; van Dissel, J. T. Antisense-mediated exon skipping to correct IL-12Rbeta1 deficiency in T cells.Blood2009,113(19), 4548-55

(8) Bestas, B.; Moreno, P. M.; Blomberg, K. E.; Mohammad, D. K.;Saleh, A. F.; Sutlu, T.; Nordin, J. Z.; Guterstam, P.; Gustafsson, M. O.;Kharazi, S.; Piatosa, B.; Roberts, T. C.; Behlke, M. A.; Wood, M. J.;Gait, M. J.; Lundin, K. E.; El Andaloussi, S.; Mansson, R.; Berglof, A.;Wengel, J.; Smith, C. I. Splice-correcting oligonucleotides restore BTKfunction in X-linked agammaglobulinemia model.J. Clin. Invest.2014,124(9), 4067-81

(9) Bauman, J. A.; Li, S. D.; Yang, A.; Huang, L.; Kole, R. Anti-tumoractivity of splice-switching oligonucleotides.Nucleic Acids Res.2010,38(22), 8348-56

(10) Zammarchi, F.; de Stanchina, E.; Bournazou, E.; Supakorndej,T.; Martires, K.; Riedel, E.; Corben, A. D.; Bromberg, J. F.; Cartegni,L. Antitumorigenic potential of STAT3 alternative splicing modu-lation.Proc. Natl. Acad. Sci. U. S. A. 2011,108(43), 17779-84

(11) Disterer, P.; Al-Shawi, R.; Ellmerich, S.; Waddington, S. N.;Owen, J. S.; Simons, J. P.; Khoo, B. Exon skipping of hepatic APOBpre-mRNA with splice-switching oligonucleotides reduces LDLcholesterol in vivo.Mol. Ther.2013,21(3), 602-9

(12) Svasti, S.; Suwanmanee, T.; Fucharoen, S.; Moulton, H. M.;Nelson, M. H.; Maeda, N.; Smithies, O.; Kole, R. RNA repair restoreshemoglobin expression in IVS2-654 thalassemic mice.Proc. Natl.Acad. Sci. U. S.

A. 2009, 106(4), 1205-10

(13) Juliano, R. L.; Ming, X.; Nakagawa, O. The chemistry andbiology of oligonucleotide conjugates.Acc. Chem. Res.2012,45(7),1067-76

(14) Sorlier, P.; Denuziere, A.; Viton, C.; Domard, A. Relationbetween the degree of acetylation and the electrostatic properties ofchitin and chitosan.Biomacromolecules2001,2(3), 765-72

(15) Buschmann, M. D.; Merzouki, A.; Lavertu, M.; Thibault, M.;Jean, M.; Darras, V. Chitosans for delivery of nucleic acids.Adv. DrugDelivery Rev.2013,65(9), 1234-70

(16) Gomes, C. P.; Ferreira Lopes, C. D.; Duarte Moreno, P. M.;Varela-Moreira, A.; Alonso, M. J.; Pêgo, A. P. Translating chitosan toclinical delivery of nucleic acid-based drugs.MRS Bull.2014,39(01),60-70 
(17) Kim, S. T.; Kim, C. K. Water-soluble chitosan-based antisenseoligodeoxynucleotide of interleukin-5 for treatment of allergic rhinitis.Biomaterials2007,28(22), 3360-8

(18) Taetz, S.; Nafee, N.; Beisner, J.; Piotrowska, K.; Baldes, C.;Murdter, T. E.; Huwer, H.; Schneider, M.; Schaefer, U. F.; Klotz, U.;Lehr, C. M. The influence of chitosan content in cationic chitosan/PLGA nanoparticles on the delivery efficiency of antisense $2^{\prime}$-O-methyl-RNA directed against telomerase in lung cancer cells. Eur. J.Pharm. Biopharm. 2009,72(2), 358-69

(19) Hong, H. J.; Jin, S. E.; Park, J. S.; Ahn, W. S.; Kim, C. K.Accelerated wound healing by smadz antisense oligonucleotides-impregnated chitosan/alginate polyelectrolyte complex.Biomaterials2008, 29(36), 4831-7

(20) Ozbas-Turan, S.; Akbuga, J.; Sezer, A. D. Topical application ofantisense oligonucleotide-loaded chitosan nanoparticles to rats. Oligonucleotides2010,20(3), 147-53

(21) Csaba, N.; Koping-Hoggard, M.; Alonso, M. J. Ionicallycrosslinked chitosan/tripolyphosphate nanoparticles for oligonucleo-tide and plasmid DNA delivery.Int. J. Pharm.2009,382(1-2), 205-14

(22) Thanou, M.; Florea, B. I.; Geldof, M.; Junginger, H. E.;Borchard, G. Quaternized chitosan oligomers as novel gene deliveryvectors in epithelial cell lines.Biomaterials2002,23(1), 153-9

(23) Liu, Z.; Zhang, Z.; Zhou, C.; Jiao, Y. Hydrophobic modificationsof cationic polymers for gene delivery.Prog. Polym. Sci.2010,35(9),1144-1162

(24) Lee, K. Y.; Kwon, I. C.; Kim, Y. H.; Jo, W. H.; Jeong, S. Y.Preparation of chitosan self-aggregates as a gene delivery system.J.Controlled Release1998,51(2-3), 213-20

(25) Chiu, Y. L.; Ho, Y. C.; Chen, Y. M.; Peng, S. F.; Ke, C. J.; Chen, K. J.; Mi, F. L.; Sung, H. W. The characteristics, cellular uptake andintracellular trafficking of nanoparticles made of hydrophobically-modified chitosan.J.

Controlled Release2010,146(1), 152-9

(26) Hu, F. Q.; Zhao, M. D.; Yuan, H.; You, J.; Du, Y. Z.; Zeng, S. Anovel chitosan oligosaccharide-stearic acid micelles for gene delivery:properties and in vitro transfection studies.Int. J. Pharm.2006,315(1-2), 158-66

(27) Noh, S. M.; Han, S. E.; Shim, G.; Lee, K. E.; Kim, C. W.; Han, S.S.; Choi, Y.; Kim, Y. K.; Kim, W. K.; Oh, Y. K. Tocopheryloligochitosan-based self assembling oligomersomes for siRNA delivery.Biomaterials2011,32(3), 849-57

(28) Brugnerotto, J.; Lizardi, J.; Goycoolea, F. M.; Arguelles-Monal,W.; Desbrieres, J.; Rinaudo, M. An infrared investigation in relationwith chitin and chitosan characterization.Polymer2001,42(8), 3569-3580

(29) Mourya, V. K.; Inamdar, N. N. Trimethyl chitosan and itsapplications in drug delivery.J. Mater. Sci.: Mater. Med.2009,20(5),1057-79

(30) Administration, U. S. F. a. D., Guidance for Industry - Pyrogen andEndotoxins Testing; Services, U. S. D. o. H. a. H., Ed. U.S. Departmentof Health and Human Services: United States, 2012

(31) Kang, S. H.; Cho, M. J.; Kole, R. Up-regulation of luciferasegene expression with antisense oligonucleotides: implications andapplications in functional assay development.Biochemistry1998,37(18), 6235-9

(32) Muzzarelli, R. A. A. Colorimetric Determination of Chitosan.Anal. Biochem.1998,26o(2), 255-257

(33) Neu, M.; Sitterberg, J.; Bakowsky, U.; Kissel, T. Stabilizednanocarriers for plasmids based upon cross-linked poly(ethyleneimine).Biomacromolecules2006,7(12), 3428-38

(34) Varkouhi, A. K.; Verheul, R. J.; Schiffelers, R. M.; Lammers, T.;Storm, G.; Hennink, W. E. Gene silencing activity of siRNA polyplexesbased on thiolated N,N,N-trimethylated chitosan.Bioconjugate Chem.2010, 21(12), $2339-46$

(35) Richard, J. P.; Melikov, K.; Vives, E.; Ramos, C.; Verbeure, B.;Gait, M. J.; Chernomordik, L. V.; Lebleu, B. Cellpenetrating peptides.A reevaluation of the mechanism of cellular uptake.J. Biol. Chem. 2002, 278(1), 585-90 
(36) Thibault, M.; Nimesh, S.; Lavertu, M.; Buschmann, M. D.Intracellular trafficking and decondensation kinetics of chitosan-pDNApolyplexes.Mol. Ther.2010,18(10), 1787-95

(37) Cheng, J.; Zeidan, R.; Mishra, S.; Liu, A.; Pun, S. H.; Kulkarni, R.P.; Jensen, G. S.; Bellocq, N. C.; Davis, M. E. Structure-functioncorrelation of chloroquine and analogues as transgene expressionenhancers in nonviral gene delivery.J. Med. Chem.2006,49(22),6522-31

(38) Hoffman, E. P.; Connor, E. M. Orphan drug development inmuscular dystrophy: update on two large clinical trials of dystrophinrescue therapies.Discov Med.2013,16(89), 233-9.

(39) Wang, M.; Wu, B.; Lu, P.; Tucker, J. D.; Milazi, S.; Shah, S. N.;Lu, Q. L. Pluronic-PEl copolymers enhance exonskipping of $2^{\prime}$-O-methyl phosphorothioate oligonucleotide in cell culture anddystrophic mdx mice.Gene Ther.2014, 21(1), 52-9

(40) Bolcato-Bellemin, A. L.; Bonnet, M. E.; Creusat, G.; Erbacher,P.; Behr, J. P. Sticky overhangs enhance siRNAmediated genesilencing.Proc. Natl. Acad. Sci. U. S. A. 2007,104(41), 16050-5

(41) Mao, H. Q.; Roy, K.; Troung-Le, V. L.; Janes, K. A.; Lin, K. Y.;Wang, Y.; August, J. T.; Leong, K. W. ChitosanDNA nanoparticles asgene carriers: synthesis, characterization and transfection efficiency.J.Controlled Release2001,70(3), 399-421

(42) Tavares, I. S.; Caroni, A. L.; Dantas Neto, A. A.; Pereira, M. R.;Fonseca, J. L. Surface charging and dimensions of chitosan coacervatednanoparticles. Colloids Surf., B2012,90, 254-8

(43) Le Tien, C.; Lacroix, M.; Ispas-Szabo, P.; Mateescu, M. A. N-acylated chitosan: hydrophobic matrices for controlled drug release.J.Controlled Release2003,93(1), 1-13

(44)Philippova,O.E.;Korchagina,E.V.Chitosananditshydrophobic derivatives: Preparation and aggregation in diluteaqueous solutions.Polym. Sci., Ser. A2012,54(7), 552-572

(45) Korchagina, E. V.; Philippova, O. E. Effects of hydrophobicsubstituents and salt on core-shell aggregates of hydrophobicallymodified chitosan: light scattering study.Langmuir2012,28(20),7880-8

(46) Wang, J.; Tao, X.; Zhang, Y.; Wei, D.; Ren, Y. Reversion ofmultidrug resistance by tumor targeted delivery of antisenseoligodeoxynucleotides in hydroxypropyl-chitosan nanoparticles.Bio-materials2010,31(15), 4426-33

(47) Gao, S.; Chen, J.; Dong, L.; Ding, Z.; Yang, Y. H.; Zhang, J.Targeting delivery of oligonucleotide and plasmid DNA to hepatocytevia galactosylated chitosan vector.Eur. J. Pharm. Biopharm. 2005,60(3), 327-34

(48) Thibault, M.; Astolfi, M.; Tran-Khanh, N.; Lavertu, M.; Darras, V.; Merzouki, A.; Buschmann, M. D. Excess polycation mediatesefficient chitosan-based gene transfer by promoting lysosomal releaseof the polyplexes.Biomaterials2011,32(20), 4639-46

(49) Holzerny, P.; Ajdini, B.; Heusermann, W.; Bruno, K.; Schuleit,M.; Meinel, L.; Keller, M. Biophysical properties of chitosan/siRNApolyplexes: profiling the polymer/siRNA interactions and bioactivity.J.Controlled Release2012,157(2), 297-304

(50) Liu, X.; Howard, K. A.; Dong, M.; Andersen, M. O.; Rahbek, U.L.; Johnsen, M. G.; Hansen, O. C.; Besenbacher, F.; Kjems, J. Theinfluence of polymeric properties on chitosan/siRNA nanoparticleformulation and gene silencing.Biomaterials2007,28(6), 1280-8

(51) Koping-Hoggard, M.; Tubulekas, I.; Guan, H.; Edwards, K.;Nilsson, M.; Varum, K. M.; Artursson, P. Chitosan as a nonviral genedelivery system. Structure-property relationships and characteristicscompared with polyethylenimine in vitro and after lung administrationin vivo.Gene Ther.2001,8(14), 1108-21

(52) Moreira, C.; Oliveira, H.; Pires, L. R.; Simoes, S.; Barbosa, M.A.; Pego, A. P. Improving chitosan-mediated gene transfer by theintroduction of intracellular buffering moieties into the chitosanbackbone.Acta Biomater.2009,5(8), 2995-3006 
(53) Li, Z.-T.; Guo, J.; Zhang, J.-S.; Zhao, Y.-P.; Lv, L.; Ding, C.;Zhang, X.-Z. Chitosan-graft-polyethylenimine with improved proper-ties as a potential gene vector.Carbohydr. Polym.2010,80(1), 254-259

(54) Pires, L. R.; Oliveira, H.; Barrias, C. C.; Sampaio, P.; Pereira, A.J.; Maiato, H.; Simoes, S.; Pego, A. P. Imidazolegrafted chitosan-mediated gene delivery: in vitro study on transfection, intracellulartrafficking and degradation.Nanomedicine (London, U. K.)2011,6(9),1499-512

(55) Lazzari, S.; Moscatelli, D.; Codari, F.; Salmona, M.; Morbidelli,M.; Diomede, L. Colloidal stability of polymeric nanoparticles inbiological fluids.J. Nanopart. Res.2012,14(6), 920

(56) Agarwal, A.; Vilensky, R.; Stockdale, A.; Talmon, Y.; Unfer, R.C.; Mallapragada, S. K. Colloidally stable novel copolymeric system forgene delivery in complete growth media.J. Controlled Release2007,121(1-2), 28-37.

(57) Buyens, K.; Meyer, M.; Wagner, E.; Demeester, J.; De Smedt, S.C.; Sanders, N. N. Monitoring the disassembly of siRNA polyplexes inserum is crucial for predicting their biological efficacy.J. ControlledRelease2010,141(1), 38-41

(58) Zhang, H.; Zhu, D.; Song, L.; Liu, L.; Dong, X.; Liu, Z.; Leng, X.Arginine conjugation affects the endocytic pathways of chitosan/DNAnanoparticles.J. Biomed. Mater. Res., Part A2011,98(2), 296-302

(59) Geary, R. S.; Wancewicz, E.; Matson, J.; Pearce, M.; Siwkowski,A.; Swayze, E.; Bennett, F. Effect of dose and plasma concentration onliver uptake and pharmacologic activity of a $2^{\prime}$-methoxyethyl modifiedchimeric antisense oligonucleotide targeting PTEN.Biochem. Pharmacol.2009,78(3), 284-91

(6o) Koller, E.; Vincent, T. M.; Chappell, A.; De, S.; Manoharan, M.;Bennett, C. F. Mechanisms of single-stranded phosphorothioatemodified antisense oligonucleotide accumulation in hepatocytes. Nucleic Acids Res.2011,39(11), 4795-807

(61) Gruber, H. J.; Hahn, C. D.; Kada, G.; Riener, C. K.; Harms, G.S.; Ahrer, W.; Dax, T. G.; Knaus, H. G. Anomalous fluorescenceenhancement of $\mathrm{Cy}_{3}$ and $\mathrm{cy}_{3} .5$ versus anomalous fluorescence loss of $\mathrm{Cy}_{5}$ and $\mathrm{Cy}_{7}$ upon covalent linking to $\lg G$ and noncovalent binding toavidin. Bioconjugate Chem. 2000,11(5), 696-704

(62) Zheng, M.; Pavan, G. M.; Neeb, M.; Schaper, A. K.; Danani, A.;Klebe, G.; Merkel, O. M.; Kissel, T. Targeting the blind spot ofpolycationic nanocarrier-based siRNA delivery.ACS Nano2012,6(11), 9447-54

(63) Van Rompaey, E.; Engelborghs, Y.; Sanders, N.; De Smedt, S.C.; Demeester, J. Interactions between oligonucleotides and cationicpolymers investigated by fluorescence correlation spectroscopy.Pharm. Res.2001,18(7), 928-36

(64) Brown, D. A.; Kang, S. H.; Gryaznov, S. M.; DeDionisio, L.;Heidenreich, O.; Sullivan, S.; Xu, X.; Nerenberg, M. I. Effect ofphosphorothioate modification of oligodeoxynucleotides on specificprotein binding.J. Biol.

Chem.1994,269(43), 26801-5

(65) Geary, R. S. Antisense oligonucleotide pharmacokinetics andmetabolism.Expert Opin. Drug Metab. Toxicol.2009,5(4), 381-91

(66) Thilo, L.; Stroud, E.; Haylett, T. Maturation of early endosomesand vesicular traffic to lysosomes in relation to membrane recycling.J.Cell Sci.1995,108(Pt 4), 1791-803

(67) Teramura, Y.; Kaneda, Y.; Totani, T.; Iwata, H. Behavior ofsynthetic polymers immobilized on a cell membrane.Biomaterials2008,29(10), 1345-55

(68) Matsuda, M.; Hatanaka, W.; Takeo, M.; Kim, C. W.; Niidome, T.; Yamamoto, T.; Kishimura, A.; Mori, T.; Katayama, Y. Shortpeptide motifs for long-lasting anchoring to the cell surface.Bioconjugate Chem.2014, 25(12), $2134-43$

(69) Hsu, C. Y.; Uludag, H. Cellular uptake pathways of lipid-modified cationic polymers in gene delivery to primary cells.Biomaterials2012,33(31), 7834-48

INSTITUTO DE INVESTİGAÇÃO E INOVAÇÃO EM SAÚDE UNIVERSIDADE DO PORTO

Rua Alfredo Allen, 208 4200-135 Porto Portugal +351220408800 
(70) Bennett, C. F.; Chiang, M. Y.; Chan, H.; Shoemaker, J. E.;Mirabelli, C. K. Cationic lipids enhance cellular uptake and activity ofphosphorothioate antisense oligonucleotides. Mol. Pharmacol.1992,41(6), 1023-33

(71) Liang, X. H.; Sun, H.; Shen, W.; Crooke, S. T. Identification andcharacterization of intracellular proteins that bind oligonucleotideswith phosphorothioate linkages. Nucleic Acids Res.2015,43, 2927. 

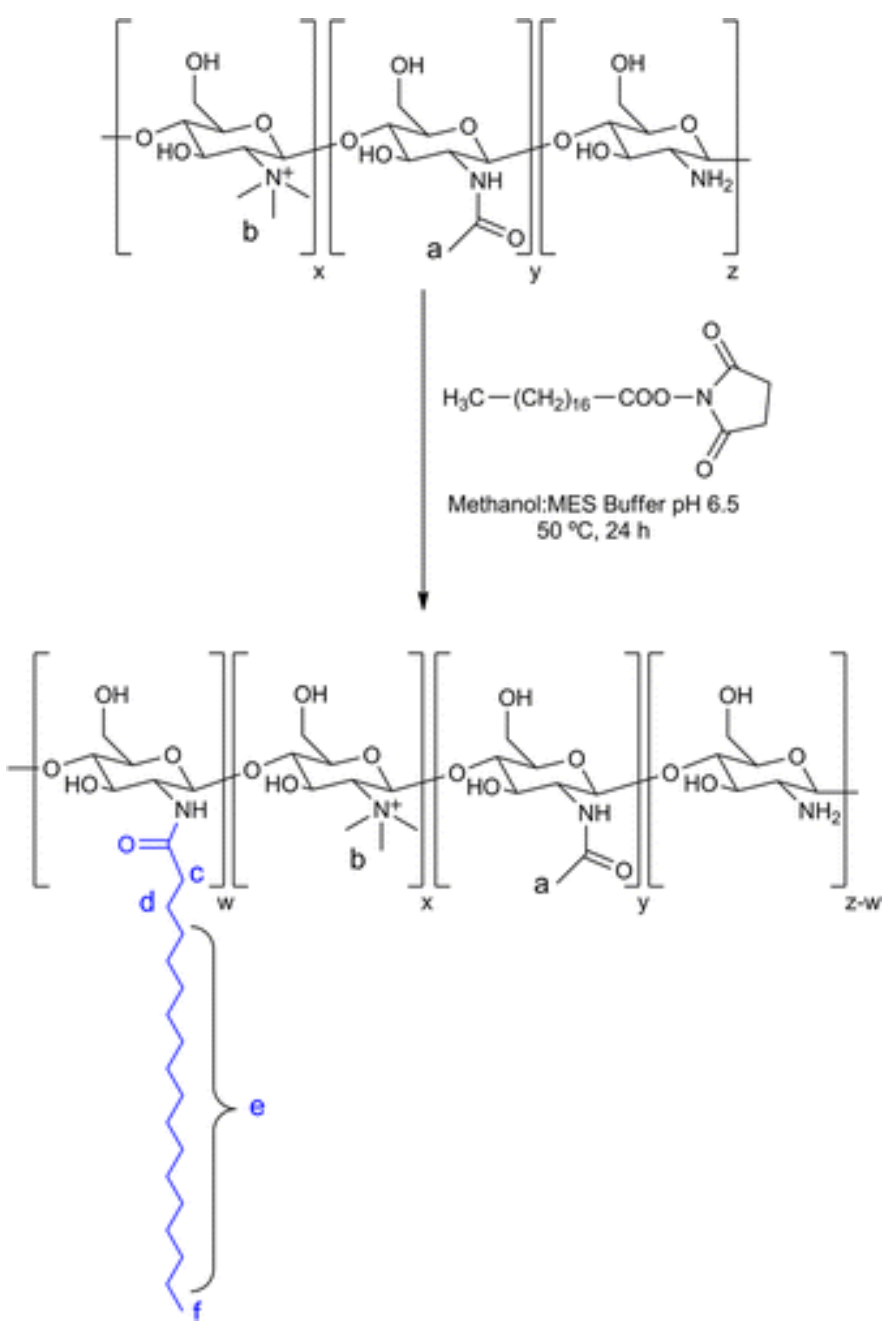

Scheme 1. Schematic Representation of Stearylated Trimethylchitosan (TMC-SA) and Synthetic Chemical Route Followed

Rua Alfredo Allen, 208 4200-135 Porto 


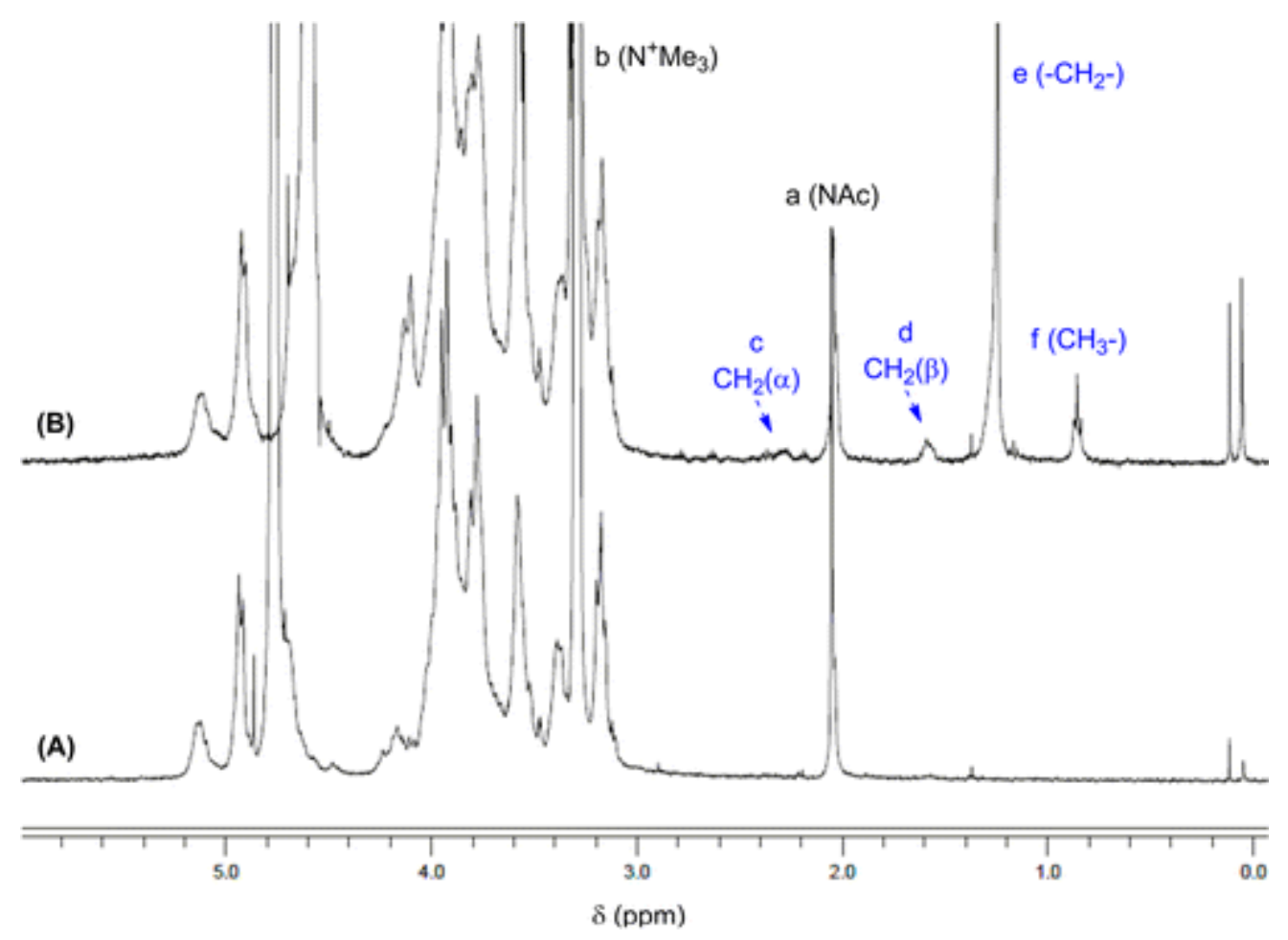

Figure 1. ${ }^{1 H}$ NMR spectra of unmodified TMC (A) and TMC-SA (B) (400 MHz, $\left.\mathrm{D}_{2} \mathrm{O}: \mathrm{MeOD}, \mathrm{DCl}\right)$. As spectra were recorded in ( $\left.\mathrm{D}_{2} \mathrm{O}: \mathrm{MeOD}: \mathrm{DCl}\right)$, the $\mathrm{MeOH}-d_{4}$ quintuplet ( $3.25 \mathrm{ppm}$ ) overlaps the $\mathrm{N}\left(\mathrm{CH}_{3}\right)_{3}$ peak $(3.24 \mathrm{ppm})$. The peaks corresponding to the grafted stearyl units, the $\mathrm{N}$-acetyl group peak, and the trimethyl group from TMC are indicated. The integrations of signals corresponding to the methyl group from stearic acid $\left(-\mathrm{CH}_{3}, \delta=0.8-0.9 \mathrm{ppm}\right)$ and the acetyl groups (NAc, $\delta=2.0-2.1 \mathrm{ppm}$ ) from TMC were used to calculate the degree of substitution (DS). 

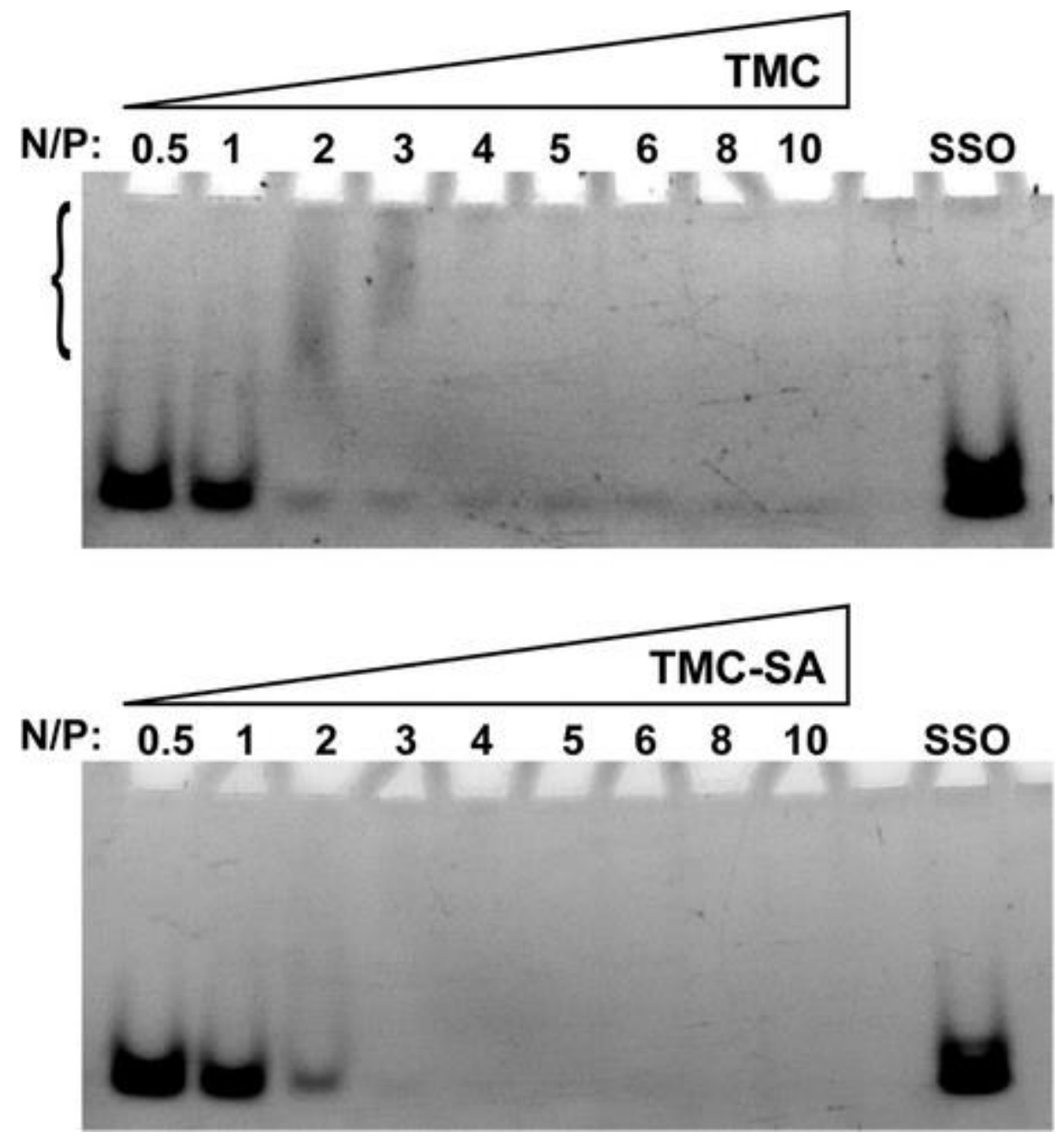

Figure 2. Gel retention assay. Polymer-SSO complexes and free SSO (control) were loaded in a 4-20\% (w/v) TBEpolyacrylamide gel, and electrophoresis was run at $140 \mathrm{~V}$. Brackets for the TMC PAGE (top gel) indicate a region of smear bands denoting delayed release of SSO. 

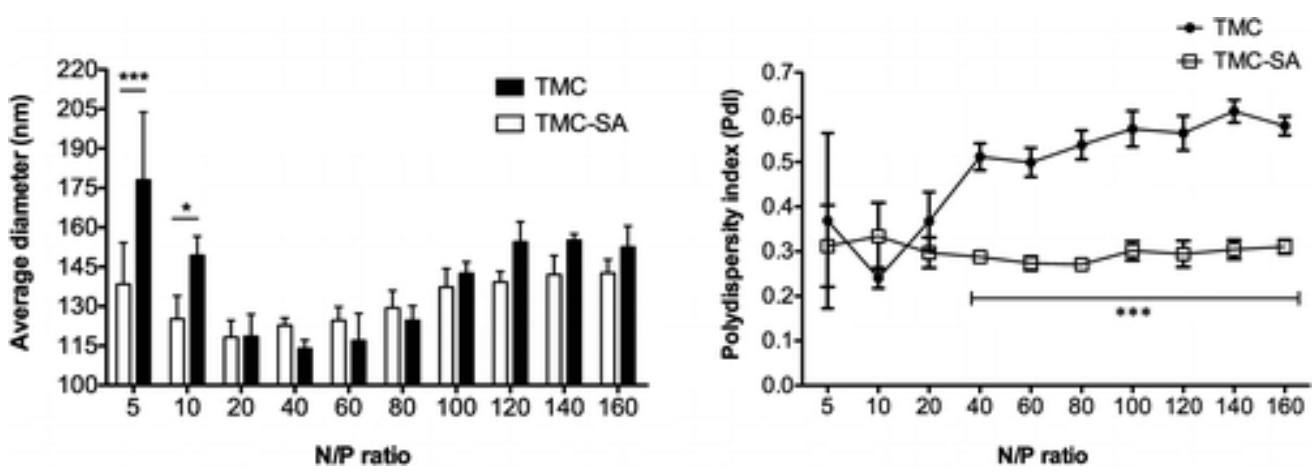

Figure 3. Characterization of polymer-SSO complexes by dynamic light scattering (size and Pdl) at different N/P ratios $(n=3$, average \pm SD). Two-way ANOVA with Bonferroni post-test was used for statistical analysis: $* * * P<$ $0.001, * * P<0.01 ;(n=3, \pm \mathrm{SD})$.

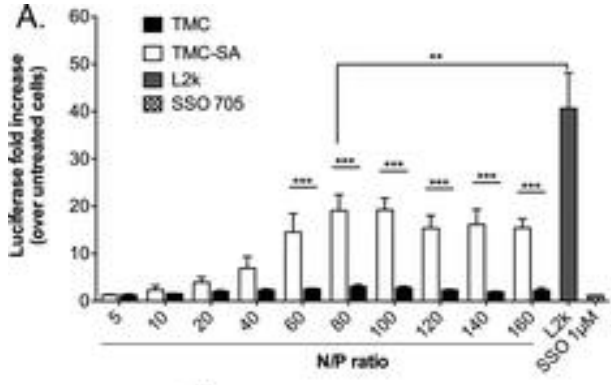

C.

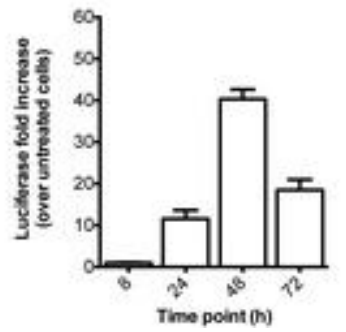

B.

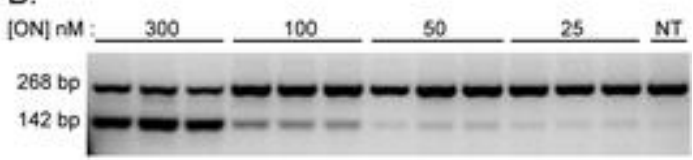

D.

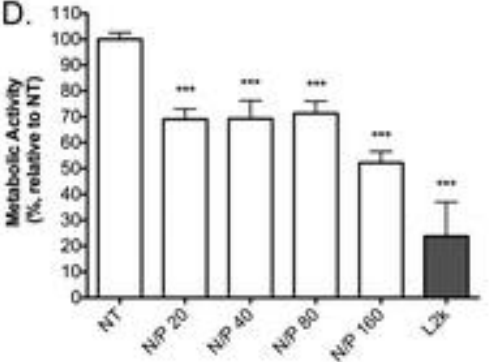

Figure 4. Splice correction activity in HeLa/Luc705 by transfection of SSO mediated by TMC-based polyplexes. Results are expressed as fold increase in luciferase activity over untreated cells. (A) Cells were incubated at $37^{\circ} \mathrm{C}$ for $24 \mathrm{~h}$ with SSO-polymer complexes at different N/P ratios, and at a fixed SSO concentration of $0.3 \mu \mathrm{M}$. Complexes were then washed out and cells continued in incubation for an additional $24 \mathrm{~h}$ before proceeding to lysis and luciferase activity determination. Additionally, as a further control, cells were incubated for $48 \mathrm{~h}$ with free $\mathrm{SSO}_{705}$ (without transfection reagents) at $1 \mu \mathrm{M}$. Two-way ANOVA with A Bonferroni post-test was used for statistical analysis: $* * * P<0.001, * * P<0.01 ;(n=3, \pm$ SD). (B) RT-PCR. Cells were incubated with SSO-polymer complexes at different SSO final concentrations, for $48 \mathrm{~h}$. Total cellular RNA was isolated and subjected to RTPCR. The upper band (268bp) and lower band (142bp) correspond to the aberrant and correct luciferase mRNA. Each lane under the corresponding concentration represents one independent experiment. (C) Cells were incubated for different periods of time with TMCSA/SSO complexes with the most effective N/P ratio of 8o, after which they were immediately analyzed for luciferase expression. Cell confluency at the day of transfection was around $50 \%$ for all time points tested in order to allow incubation up to the $72 \mathrm{~h}$ time point while maintaining equal conditions at start of transfection between the time points. (D) Cytotoxicity assay as evaluated by metabolic activity determination through the resazurin assay. TMC-SA/SSO complexes were incubated with HeLa/Luc705

INSTITUTO DE INVESTİGAÇÃO E INOVAÇÃO EM SAÚDE UNIVERSIDADE DO PORTO

Rua Alfredo Allen, 208 4200-135 Porto Portugal +351220 408800 
cells for $24 \mathrm{~h}$, at a final SSO concentration of $0.3 \mu \mathrm{M}$. Data is presented as percentage of metabolic activity relative to nontreated cells. One-way ANOVA with Dunnett's multiple comparison test (vs nontreated cells, NT) was used for statistical analysis $(n=3$, average \pm SD).
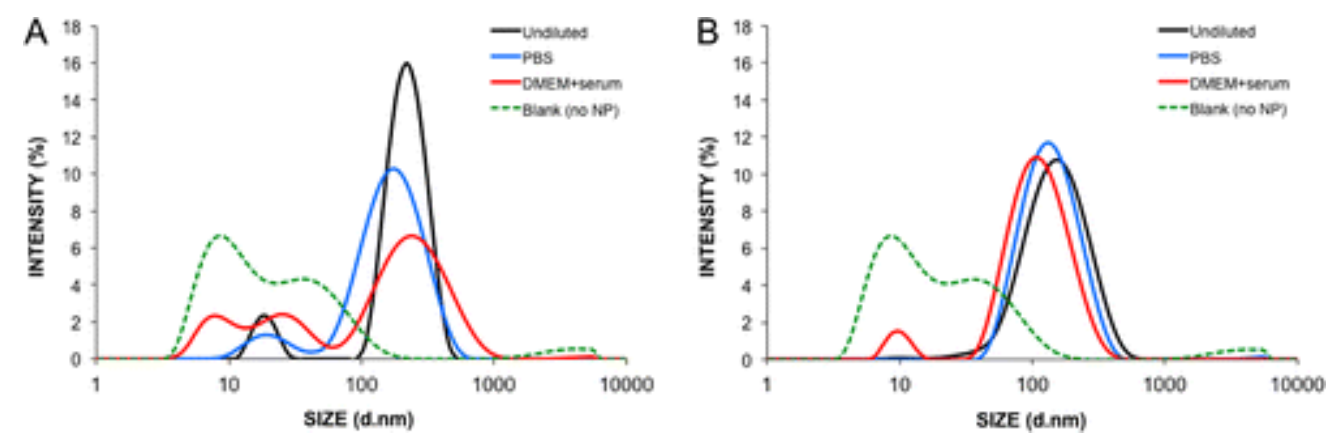

Figure 5. Stability of SSO/polymer complexes in different media. (A) TMC/SSO; (B) TMC-SA/SSO. Complexes were formed at N/P ratio 80 , diluted 3 -fold in either complexation buffer, PBS (final $1 \times$ PBS) or DMEM with $10 \%$ serum, and incubated for $1 \mathrm{~h}$ at $37^{\circ} \mathrm{C}$. Average size was then determined by DLS. Profile of DMEM with serum and no nanoparticles (Blank (no NP)) was taken in order to distinguish protein-related aggregates.

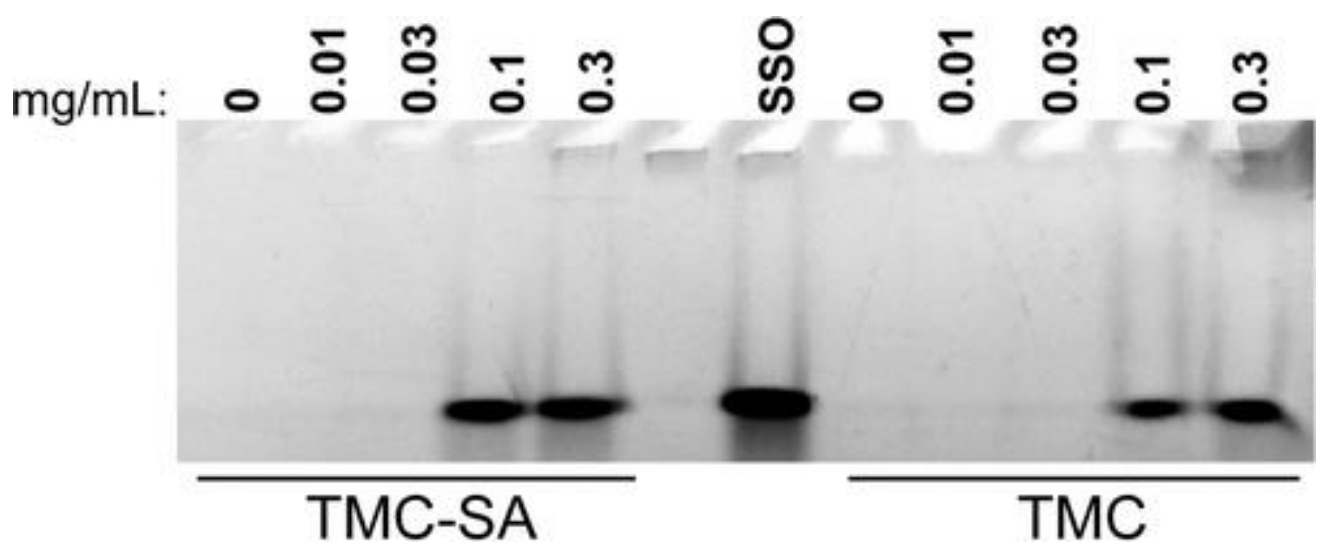

Figure 6. Heparin dissociation assay. Complexes formed between TMC and TMC-SA with SSOs, using a N/P ratio of 80 , were incubated with increasing concentrations of heparin at $37^{\circ} \mathrm{C}$ in physiological salt and $\mathrm{pH}$ conditions for $2 \mathrm{~h}$. Samples were then run using PAGE to verify the extent of dissociated SSOs from the complexes. In the SSO lane the same amount of free oligo as occurring in the nanocomplexes was loaded. 

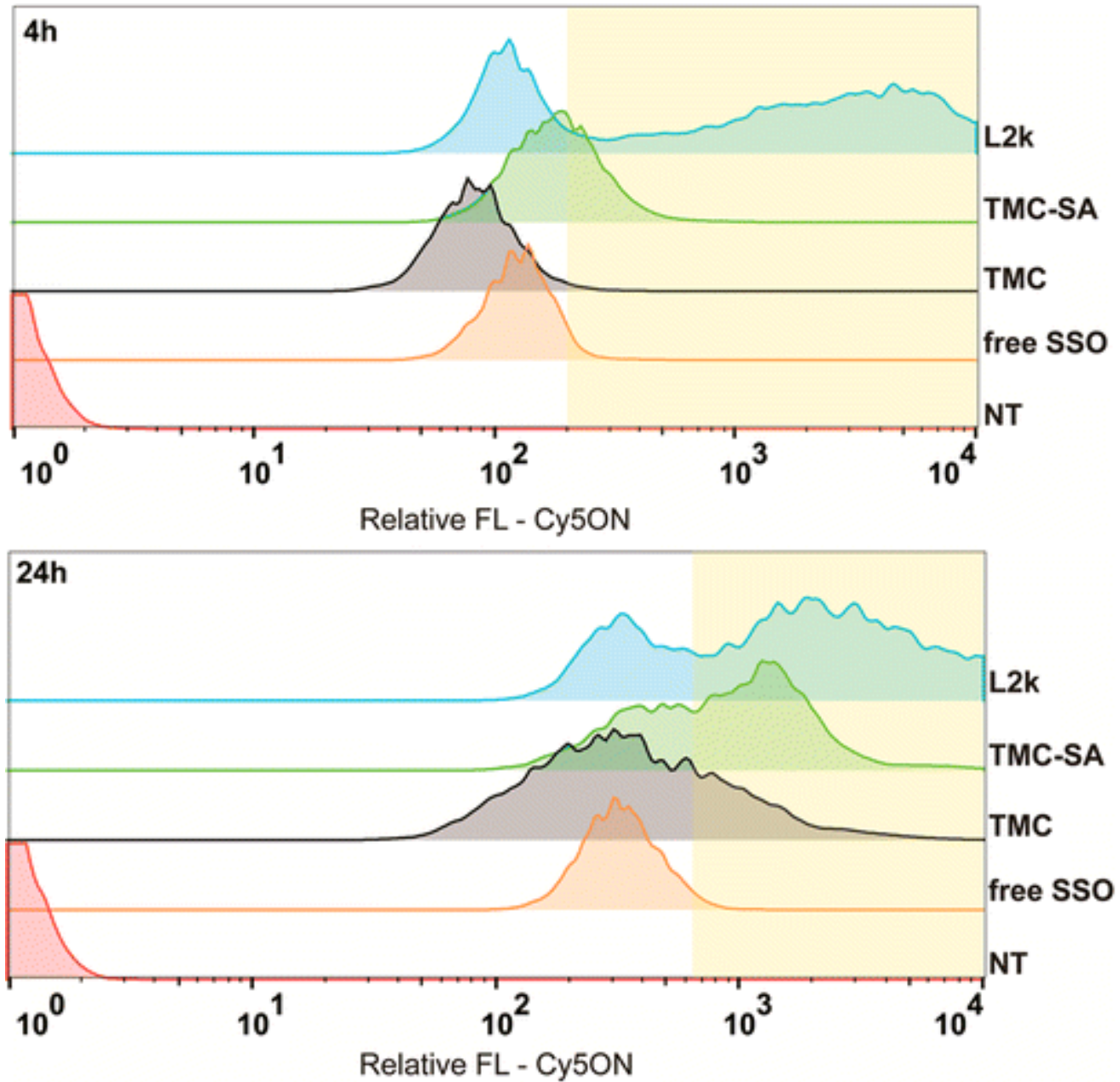

Figure 7. Characterization of cellular association of SSO/polymer complexes by flow cytometry. Complexes containing $\mathrm{Cy}_{5}$ labeled SSO (Cy5-SSO705) were incubated for 4 and $24 \mathrm{~h}$ with HeLa/Luc70 5 cells at a final SSO concentration of $0.3 \mu \mathrm{M}$. Highlighted area corresponds to populations of cells with high relative fluorescence (FL) (above free SSO), which can be attributed to the release of SSO from vesicles and dissociation from polymer. 

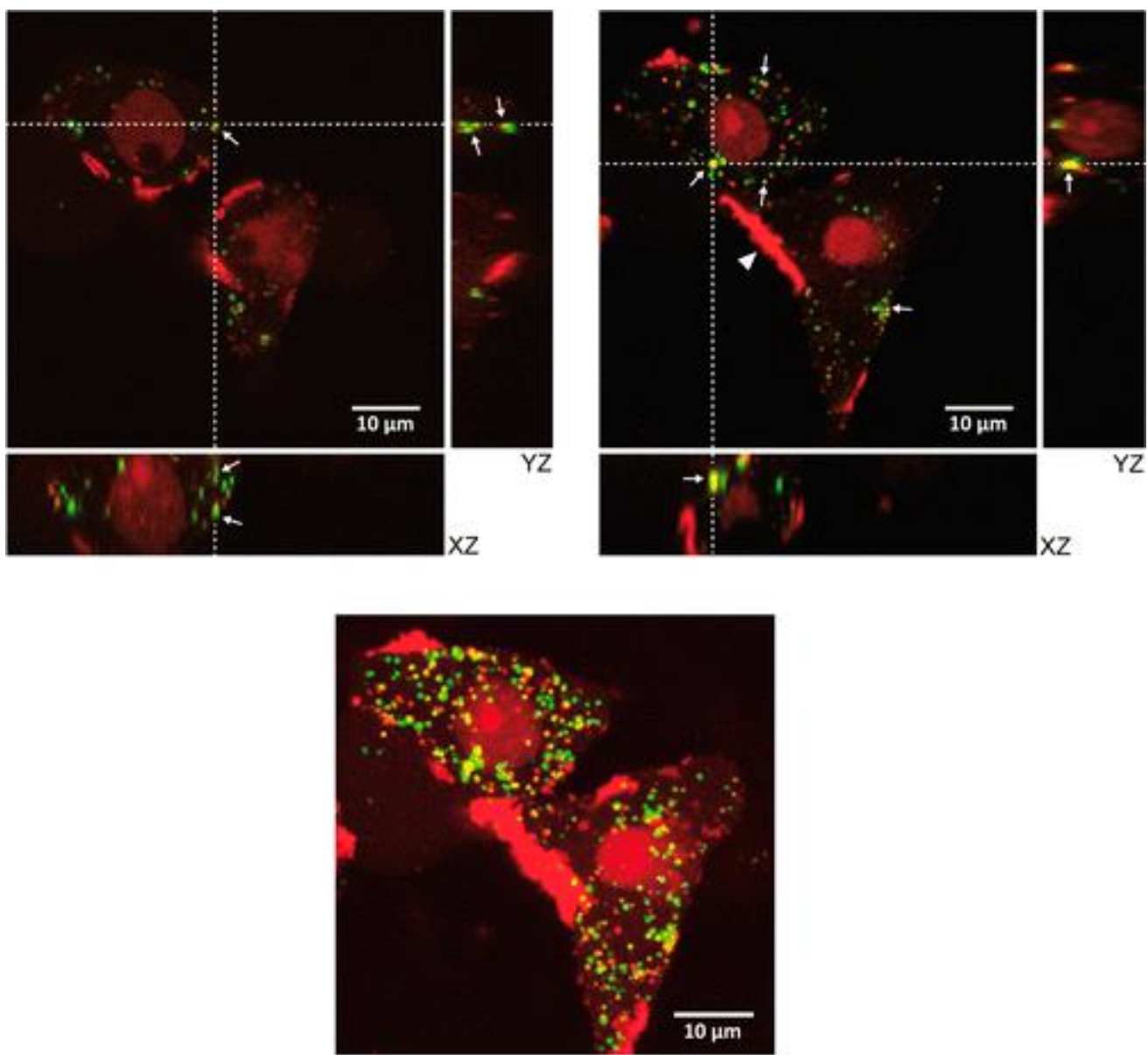

Figure 8. Live cell imaging by confocal laser scanning microscopy. TMC-SA/Cy5-SSO complexes (represented in red) at N/P 80 and $70 \mathrm{kDa}$ Rhodamine-Dextran (represented in green) were coincubated with HeLa/Luc705 cells for $24 \mathrm{~h}$. After extensive washing with PBS, OPTIMem (no phenol red) was added to the wells and cells were imaged. The two orthogonal view images (top) of the same cell represent different xyz coordinates (dashed lines indicate the $x y, x z, y z$ planes of view). Arrows point to examples of colocalization of dextran and Cy5-SSO (identified by appearance of yellow color). Arrowhead points to a region of accumulation of TMC-SA/Cy5-SSO complexes at the cell membrane. The lower image represents a maximum intensity z-projection of 15 slices, giving an overview of the vesicle spread throughout the cell and the aggregation of $\mathrm{Cy}_{5}-\mathrm{SSO}$ polyplexes at the periphery of the cell. Colocalization spots, in yellow, are present in high amounts, as observed in the z-projection and confirmed through the orthogonal view analysis. 


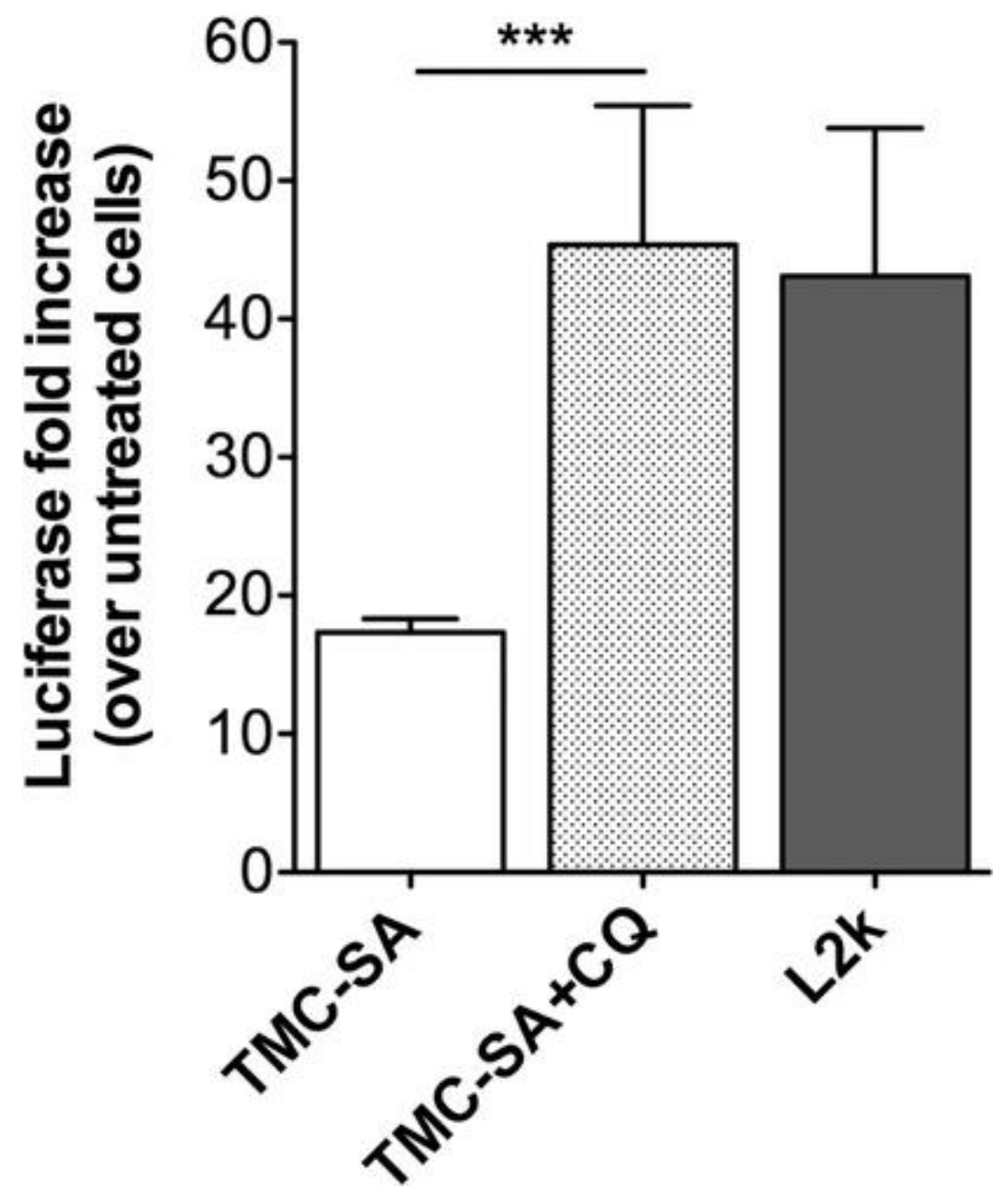

Figure 9. Cells were incubated with TMC-SA/SSO complexes at N/P ratio 80 for $24 \mathrm{~h}$, after which chloroquine (CO) was added at a final concentration of $100 \mu \mathrm{M}$ and incubated for $4 \mathrm{~h}$. Cell medium was then exchanged with fresh medium and cells finally incubated for $20 \mathrm{~h}$, giving a total of $48 \mathrm{~h}$, post-transfection, before proceeding to luciferase activity determination. One-way ANOVA with Bonferroni post-test was used for statistical analysis: *** $P<0.001 ;(n=3$, average $\pm \mathrm{SD})$. 
Table 1. Zeta Potential Measured by DLS $(n=3$, average \pm SD) a

\section{Zeta Potential (mV)}

NP (HEPES)

$8.9 \pm 1.5$

$7 \cdot 3 \pm 1.2$
NP (DMEM/FBS)

$3.8 \pm 0.7$

$2.3 \pm 0.4$
Free polymer (HEPES)

$19.6 \pm 2.1$

$18.0 \pm 2.8$

${ }^{\text {aF }}$ Free polymer (no complexation) or polymer/SSO nanoparticles (NP) formed at N/P ratio of 80 were diluted 3 -fold in the respective media.

Rua Alfredo Allen, 208 4200-135 Porto 
Supporting Information

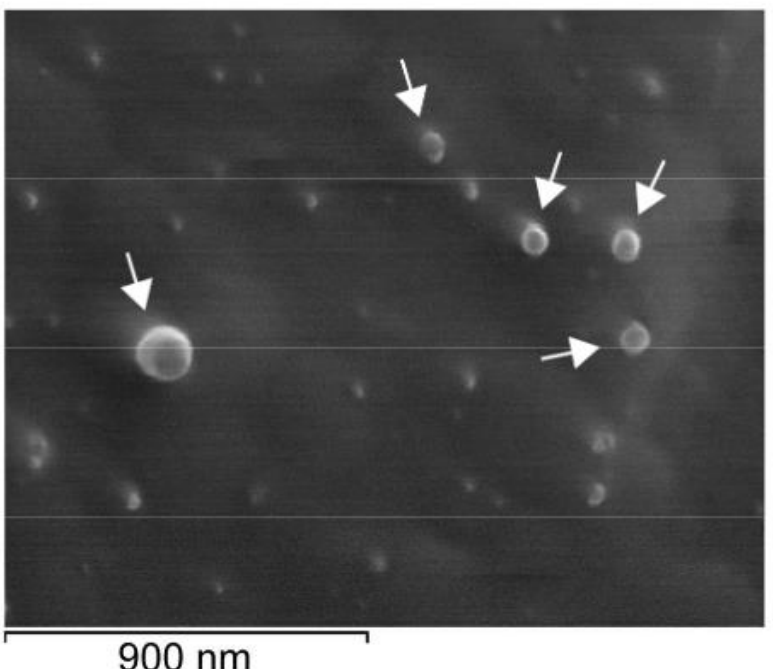

Supplementary Figure 1. Cryo-SEM image of transfection competent TMC-SA/SSO polyplexes at N/P ratio 80. White arrows indicate polyplexes. A mostly regular spherical morphology can be observed

Rua Alfredo Allen, 208 4200-135 Porto 

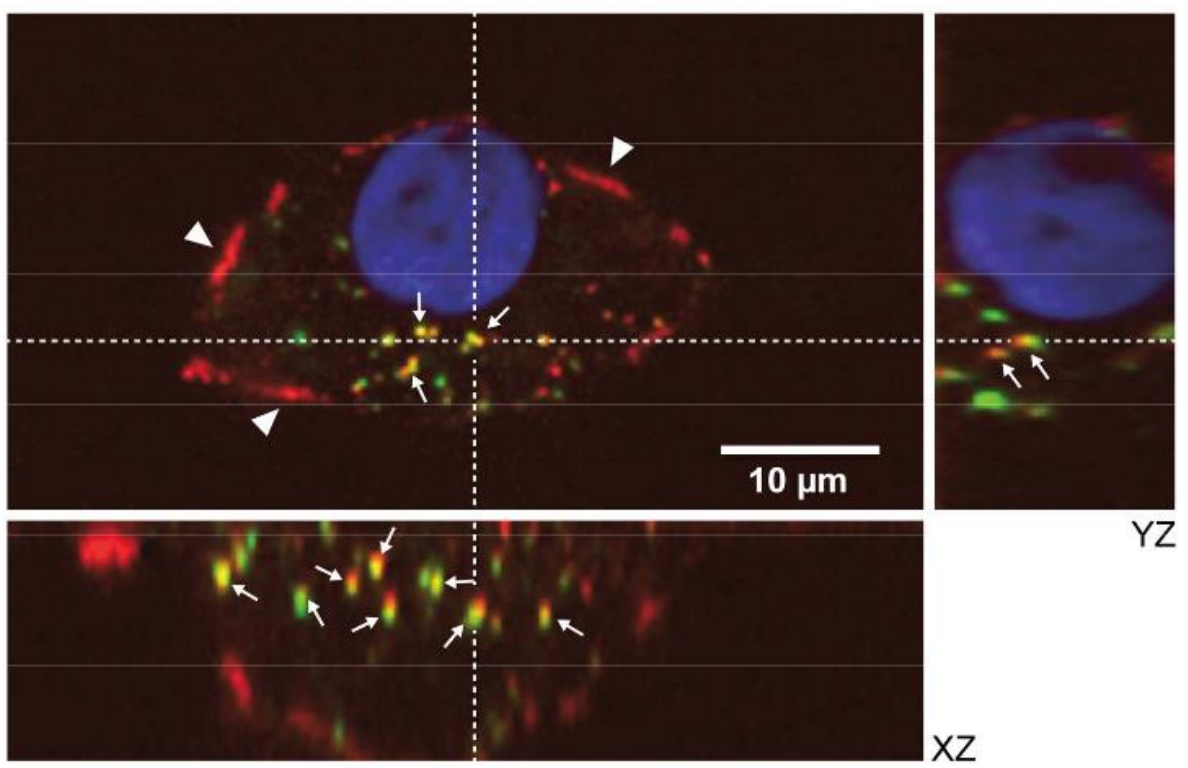

YZ

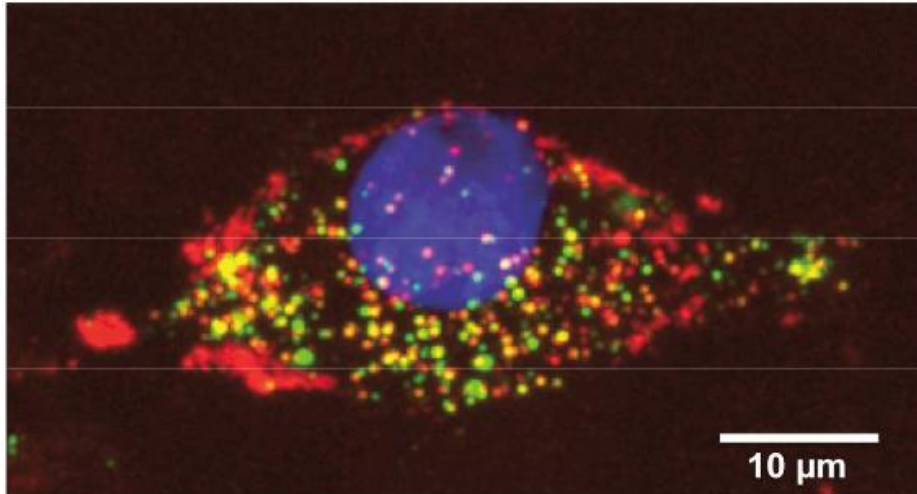

Supplementary Figure 2. Live cell imaging by confocal laser scanning microscopy. TMC-SA/Cy5-SSO complexes (represented in red) at N/P 80 and $70 \mathrm{kDa}$ Rhodamine-Dextran (represented in green) were co-incubated with HeLa/Luc705 cells for $4 \mathrm{~h}$. After extensive washing with PBS, OPTI-MEM (no phenol red) was added to the wells and cells were imaged. HOECHST (represented in blue) was used for counterstaining the nuclei. Top image represents an orthogonal view with dashed lines indicating the $x y, x z, y z$ planes of view. Arrows point to examples of co-localization of dextran and $\mathrm{Cy}_{5}$-SSO (identified by appearance of yellow color). Arrowheads point to regions of accumulation of TMC-SA/Cy5-SSO complexes at the cell membrane. The lower image represents a maximum intensity z-projection of 15 slices giving an overview of the vesicle spread throughout the cell and the aggregation of $\mathrm{Cy}_{5}$-SSO polyplexes at the periphery of the cell. Co-localization spots, in yellow, are present in high amounts as observed in the z-projection and confirmed through the orthogonal view analysis. 

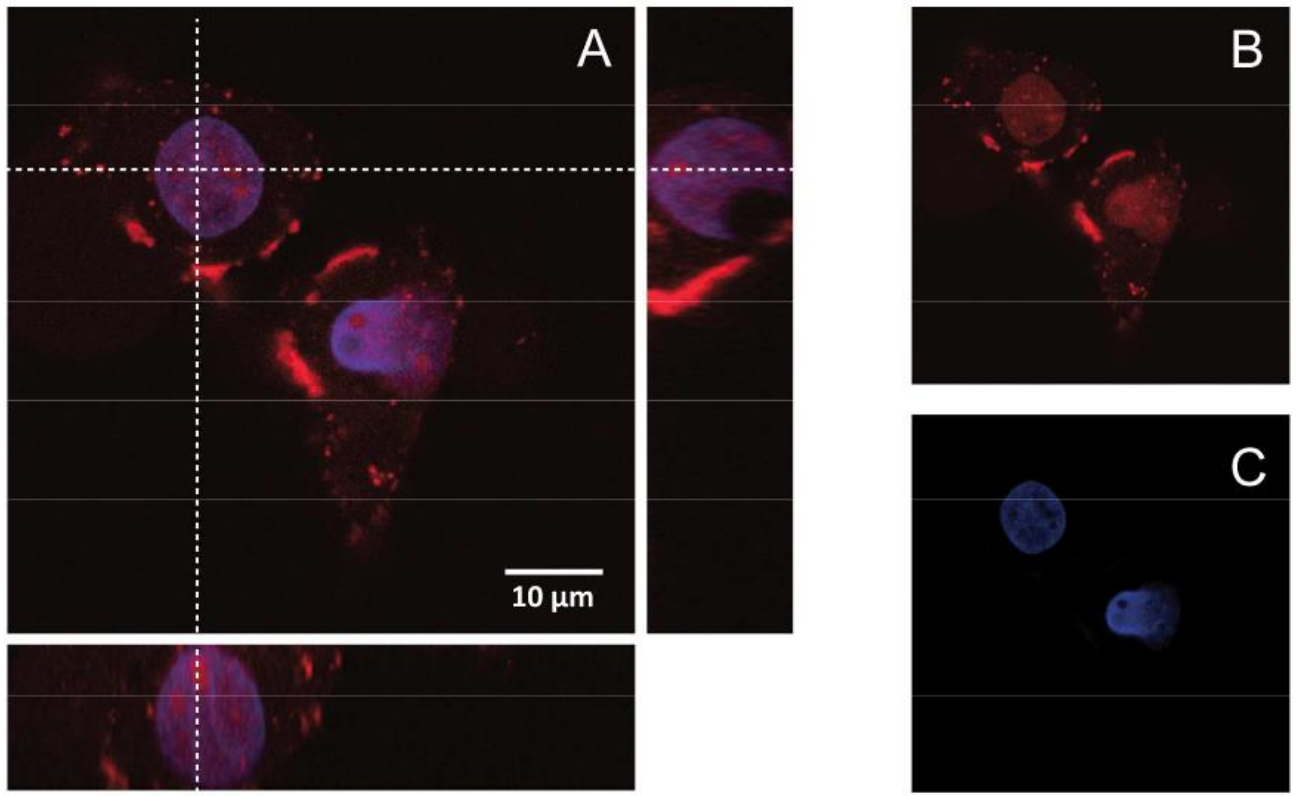

Supplementary Figure 3. View of Figure 1 focused on the nuclear (Hoechst staining, Blue) and Cy5-SSO (Red) fluorescence co-localization. A) Orthogonal views of the merged fluorescence channels. B) TMC-SA/Cy5-SSO fluorescence channel image. C) Hoechst fluorescence channel image 